\title{
Loss of WSTF results in spontaneous fluctuations of heterochromatin formation and resolution, combined with substantial changes to gene expression
}

\author{
Ashley E Culver-Cochran and Brian P Chadwick
}

\begin{abstract}
Background: Williams syndrome transcription factor (WSTF) is a multifaceted protein that is involved in several nuclear processes, including replication, transcription, and the DNA damage response. WSTF participates in a chromatin-remodeling complex with the ISWI ATPase, SNF2H, and is thought to contribute to the maintenance of heterochromatin, including at the human inactive $X$ chromosome (Xi). WSTF is encoded by BAZ1B, and is one of twenty-eight genes that are hemizygously deleted in the genetic disorder Williams-Beuren syndrome (WBS).

Results: To explore the function of WSTF, we performed zinc finger nuclease-assisted targeting of the BAZ1B gene and isolated several independent knockout clones in human cells. Our results show that, while heterochromatin at the $\mathrm{Xi}$ is unaltered, new inappropriate areas of heterochromatin spontaneously form and resolve throughout the nucleus, appearing as large DAPI-dense staining blocks, defined by histone H3 lysine-9 trimethylation and association of the proteins heterochromatin protein 1 and structural maintenance of chromosomes flexible hinge domain containing 1. In three independent mutants, the expression of a large number of genes were impacted, both up and down, by WSTF loss.

Conclusions: Given the inappropriate appearance of regions of heterochromatin in $B A Z 1 B$ knockout cells, it is evident that WSTF performs a critical role in maintaining chromatin and transcriptional states, a property that is likely compromised by WSTF haploinsufficiency in WBS patients.
\end{abstract}

\section{Background}

$\mathrm{X}$ chromosome inactivation $\mathrm{XCI}$ ) is the process whereby females balance the levels of X-linked gene expression with males [1], and is an archetypal example of epigenetic regulation. Much is known about the early stages of XCI, including counting, choice, initiation, and spreading of the inactivation signal [2], but our understanding of how chromatin of the chosen inactive $\mathrm{X}$ chromosome (Xi) and gene silencing is faithfully maintained throughout subsequent somatic cell divisions is less well understood.

The human $\mathrm{Xi}$ is primarily composed of two spatially distinct types of heterochromatin [3]; one is characterized by histone $\mathrm{H} 3$ trimethylated at lysine 9 (H3K9me3) $[4,5]$ and association of heterochromatin protein 1 (HP1) [6], whereas the other is defined by histone H3

\footnotetext{
* Correspondence: chadwick@bio.fsu.edu

Department of Biological Science, Florida State University, Tallahassee, FL, USA
}

trimethylated at lysine 27 (H3K27me3) [7,8], elevated levels of the histone variant macroH2A [9-11], and association of the X-inactive specific transcript (XIST) [12-14]. We have previously shown that the WSTF-ISWI chromatin remodeling complex (WICH) transiently associates with the human $\mathrm{Xi}$ as the chromosome is undergoing DNA replication [15] and, therefore, is a candidate for maintaining this chromatin organization. Current models suggest that Williams syndrome transcription factor (WSTF), one of two subunits in the WICH chromatin remodeling complex [16], assists in reforming chromatin states of the parental cell post DNA replication [17]. Consistent with this model, depletion of WSTF protein levels by RNA-interference (RNAi) resulted in aberrant heterochromatin formation [18].

WSTF is encoded by the gene $B A Z 1 B$, which is located at 7q11.23 [GenBank: NG027679] [19]. It is a 
multifaceted protein with ties to several cellular processes, including transcription [20], replication [16], and the DNA damage response [21]. In addition to the WICH complex, WSTF has been identified as a participant in the B-WICH complex, which is associated with transcription of the ribosomal genes [22]. WSTF is also linked to the DNA damage response pathway, and was recently shown to possess tyrosine kinase activity, phosphorylating Tyr142 of H2A.X [21]. The significance of this covalent histone modification sits at the juncture between deciding on DNA repair or apoptosis [23] based on whether the modification is removed to allow access to the MDC1 complex or retained resulting in activation of the JNK pathway [24].

Interestingly, WSTF is deficient in Williams-Beuren syndrome (WBS; OMIM\#194050), an autosomal dominant genetic disorder that affects approximately 1 in 7,500 individuals [25]. The disorder is characterized by a 1.5-1.8 megabase $(\mathrm{Mb})$ deletion from one copy of chromosome 7q11.23 [26]. The deletion arises de novo, due to low-copy repeat elements flanking either side of the break points [27], and comprises approximately twenty-eight genes [28-31]. WBS patients experience an array of symptoms including cardiac defects, cognitive impairment, hypercalcaemia, growth deficiencies, and a distinct craniofacial phenotype [32-34]. Stemming from studies performed in mouse and cell lines, WSTF has been implicated as a key contributor to many of the phenotypes seen in WBS patients, despite the number of other genes that are also deficient $[16,35,36]$. It is unclear, however, which specific gene deficiencies contribute to each symptom; a problem common to all contiguous gene deletion disorders.

The aim of this study was to investigate the impact of WSTF loss on the maintenance of chromatin at the human $\mathrm{Xi}$, and more broadly, to develop a better understanding of how the loss of this multifaceted protein affects general cellular processes. Towards this goal, we disrupted the $B A Z 1 B$ gene using zinc-finger nucleaseassisted gene targeting in the cell line hTERT-RPE1 (RPE1) - a telomerase immortalized [37], diploid female cell line-and isolated several independent clones that lack the WSTF protein. Findings from this study underscore the necessity of determining the role of WSTF, particularly in regards to chromatin maintenance.

\section{Results}

\section{Disruption of the BAZ1B gene using zinc finger nuclease technology}

The development of zinc finger nuclease technology provides opportunities for genomic editing at precise locations in human cells [38]. Here, we introduced into RPE1 cells a pair of zinc finger nucleases (ZFNs) designed to exon-7 of the $B A Z 1 B$ gene, along with an engineered repair template (Figure 1a). The repair template consisted of a central promoterless neomycin open reading frame (ORF) that was preceded by a splice acceptor and internal ribosome entry site, and followed by a polyadenylation signal [39]. This central cassette was flanked by homology arms (HA) of approximately 600-800 bp that were derived from sequences in the vicinity of exon-7 (Figure 1a). The general strategy is that upon generation of a double-stranded break by the ZFNs, the repair template is used though homology mediated repair to patch the damage, introducing the neomycin ORF in place of exon-7 in the process. This synthetic exon promoter trap (SEPT) is incorporated into the targeted $B A Z 1 B$ transcript, prematurely truncating the message through poly-adenylation and providing neomycin resistance, driven by the gene's own expression. Transfected cells were placed under neomycin selection, and after two weeks clones emerged.

Screening for correct targeting was achieved by performing PCR for both the left and right sides of the targeted region with a primer within the SEPT cassette and a primer located outside of the HAs at the genomic locus (Figure 1b). We screened a total of 119 single cell clones by PCR. We identified 13 clones that were correctly targeted at one or both alleles of the $B A Z 1 B$ gene. To screen for targeting at both alleles, a second PCR was performed with primers flanking the ZFN target site (Figure 1a). If a clone was targeted at both alleles, this sequence would no longer be present and no product would be detected. Three clones were determined to be successfully targeted at both alleles. Double-strand breaks generated by ZFNs can be repaired by either homology mediated repair or non-homologous end joining (NHEJ). NHEJ is not a precise repair pathway, frequently resulting in introduction of small insertions or deletions at the target site [40], which can disrupt the reading frame of the target gene. For those clones that were targeted at only one allele, we screened for NHEJ at the second allele by sequencing the PCR product. We identified three clones that were targeted at one allele and have the second allele disrupted by NHEJ (Figure 1c), and several clones that were targeted at one allele but wild-type at the second allele. This latter group of heterozygous mutant clones are useful models to investigate the contribution of $B A Z 1 B$ hemizygosity in WBS in the absence of the other $>20$ genes that are typically lost alongside $B A Z 1 B$ in this disease, all of which potentially contribute to the phenotype. The clones used in this study and the nature of their mutation/s is indicated in Table 1.

Quantitative real time PCR (qRT-PCR) using primers that spanned exons- 2 and -3 indicated that a clone targeted at only one allele (Clone A6), showed $B A Z 1 B$ mRNA at $47.6 \%$ of parental cells. Clone M1, targeted at one allele and mutated at the second by NHEJ, expressed 
(a) locus

Targeting

construct

(c)
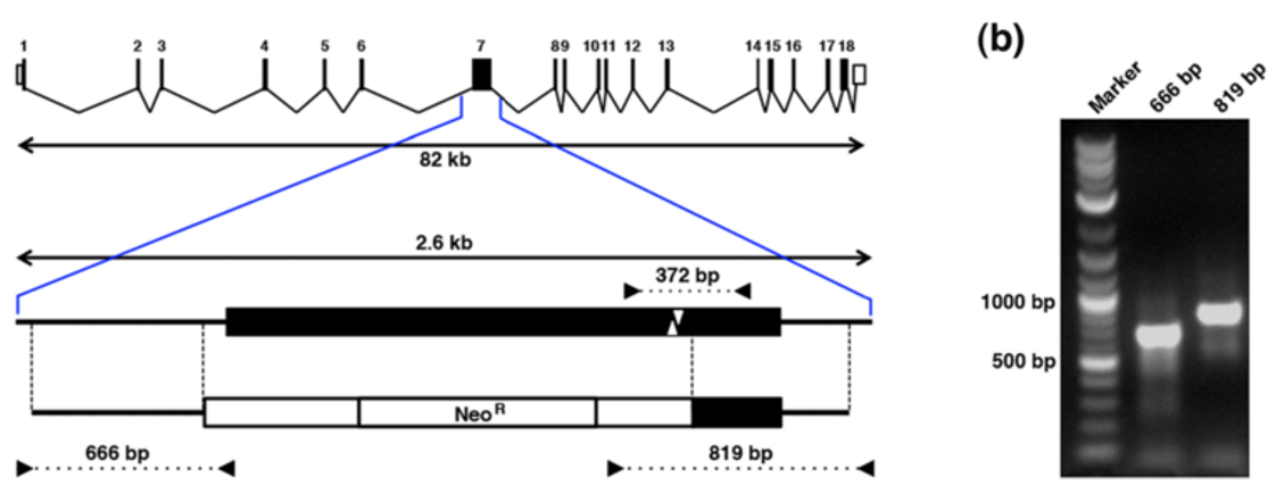

RPE1 parental GTGCCACCGGATCCTCATGACATACTCAGTGCAAGACCACATGGAGACCAGACAGCAG (16bp deletion GTGCCACCGGATCCTCATGAC 16bp CACATGGAGACCAGACAGCAG ${ }_{\text {NHEJ }}^{B A Z 1 B}$ 2bp deletion GTGCCACCGGATCCTCAT 2bp CATACTCAGTGCAAGACCACATGGAGACCAGACAGCAG NHEJ 4bp insertion GTGCCACCGGATCCTCATGACATACTCAGTGCAAGACCACATGGAGACCAGACAGCAG

(d)

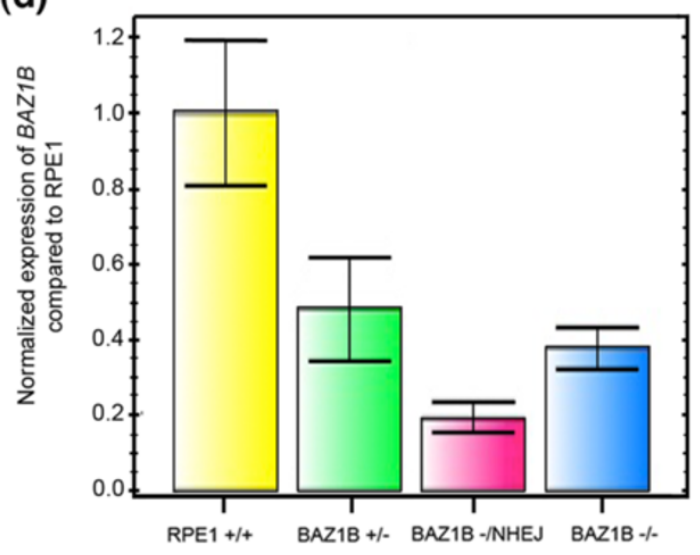

(f)
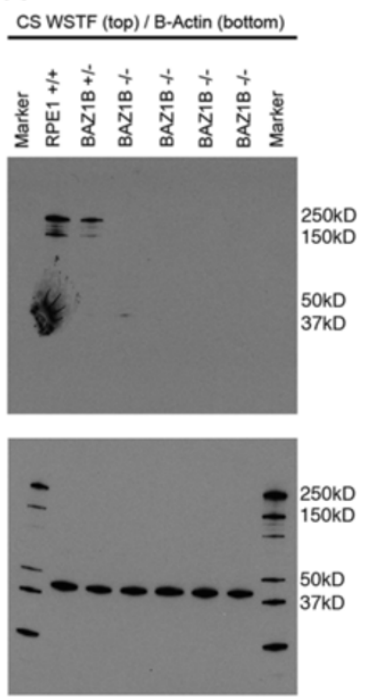

(g)

RPE1+/+

(e) $\bigwedge_{4 \mathrm{bp}}$
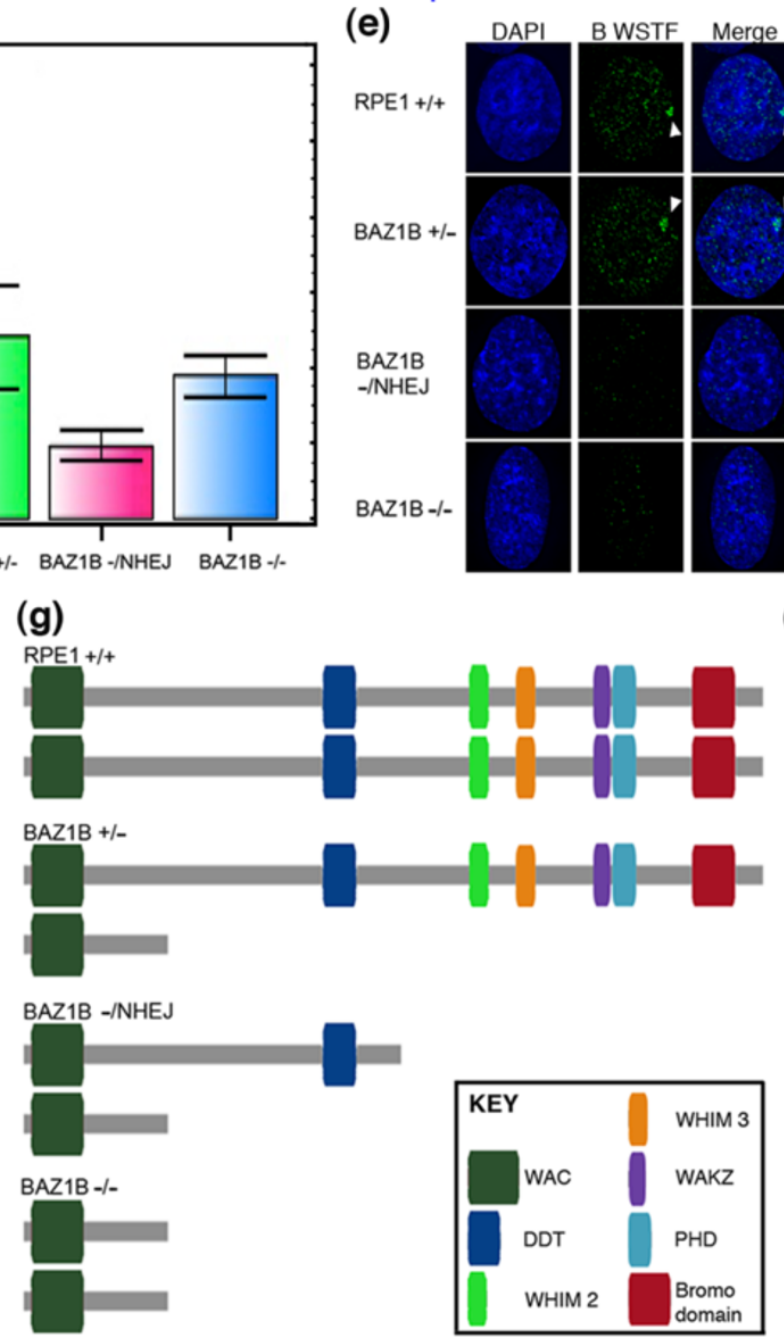

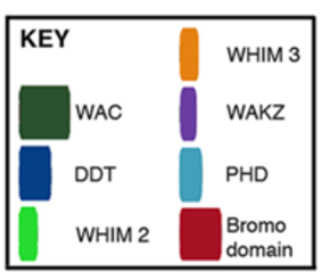

(h)
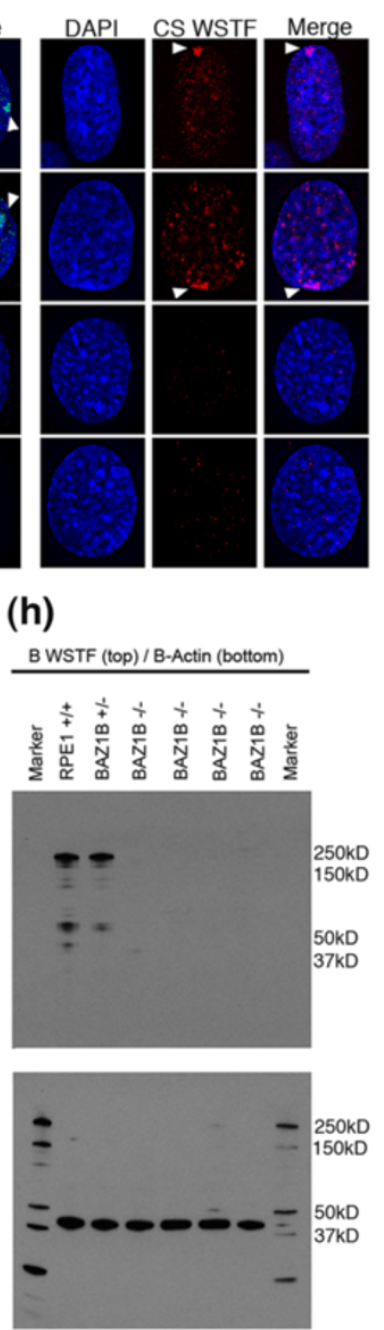

Figure 1 (See legend on next page.) 
Figure 1 Generation of $B A Z 1 B$ knockout cells. (a) Schematic representation of the BAZ1B gene. Exons: numbered vertical bars. Introns: connecting lines. Exon 7 is bracketed by the blue lines, and expanded beneath. The targeting construct is shown beneath, indicating the location of the neomycin resistance gene and the extent of the homology arms (regions between the vertical dotted lines). Left and right side screening PCRs are indicated below by inward-facing arrowheads. The NHEJ PCR is shown above Exon 7, and the ZFN cutting site is indicated by the white arrowheads within the exon. (b) Example of an ethidium bromide stained gel showing a positive clone by PCR. (c) DNA sequence alignment of parental RPE1 with NHEJ disrupted clones. The cutting site for the ZFNs is highlighted in red. Deletions in NHEJ clones are indicated by the red gaps; insertions by the blue triangle. (d) qRT-PCR analysis of BAZ1B showing levels in a heterozygous mutant (BAZ1B $+/-)$, a clone with one allele targeted and one disrupted by NHEJ (BAZ1B -/NHEJ) and a clone targeted at both alleles (BAZ1B-/-) relative to parental RPE1. (e) Indirect immunofluorescence showing the distribution of WSTF in RPE1, a BAZ1B +/-, BAZ1B -/NHEJ and a BAZ1B -/- clone. WSTF is shown in green for the Bethyl Labs anti-WSTF antibody and red for the Cell Signaling anti-WSTF antibody. The white arrowheads indicate the location of the Xi. The nucleus is counterstained with DAPI (blue). (f) Western blot for WSTF with the Cell Signaling antibody in RPE1, a BAZ1B $+/-$, and various independent BAZ1B knockout mutants. Actin is shown immediately below. (g) Schematic map of WSTF showing the relative location of functional domains, and the truncated proteins possible in the different mutant types. (h) Western blot using the Bethyl Labs anti-WSTF antibody as described for part-f above.

$B A Z 1 B$ mRNA at $18.3 \%$, whereas clone D5, that is targeted at both alleles, showed 33.7\% (Figure 1d). Expression of $B A Z 1 B$ is expected as exons 1-6 are uninterrupted and isolation of clones required $B A Z 1 B$ expression to trap the SEPT cassette and express neomycin resistance. The reduced levels of mRNA likely represent destabilization of the BAZ1B mRNA.

We then employed indirect immunofluorescence (IF) to investigate the distribution of WSTF in the $B A Z 1 B$ mutants. Here, we used two independent antibodies raised to different regions of the WSTF protein; one to the first 50 amino acids, and the other to a peptide at the carboxy terminus. Results indicate that WSTF distribution in the $B A Z 1 B$ heterozygous mutant was very similar to that of the parental cells, whereas WSTF staining was absent in all double-targeted clones suggesting a complete knockout of the gene (Figure 1e). To confirm loss of WSTF, Western blotting was performed on protein extracts from the mutants. Consistent with the IF analysis, protein levels were reduced in the heterozygous mutant and absent from all independent homozygous mutant clones (Figure 1f). By targeting exon-7, the ORF contained within the first 6 exons could potentially generate a truncated protein of $87.3 \mathrm{kDa}$ (from NHEJ targeted alleles, amino acids 1-761) or $34.5 \mathrm{kDa}$ (from double targeted alleles, amino acids 1-

Table 1 BAZ1B mutations in clones used in this study

\begin{tabular}{ccc}
\hline Clone & BAZ1B Allele 1 & BAZ1B Allele 2 \\
\hline BAZ1B-A6 (+/-) & Promoter trap targeted & Wild type \\
BAZ1B-D5 (-/-) & Promoter trap targeted & Promoter trap targeted \\
BAZ1B-F3 $(-/-)$ & Promoter trap targeted & Promoter trap targeted \\
BAZ1B-M1 (-/-) & Promoter trap targeted & 16 bp deletion \\
BAZ1B-N6 (-/-) & Promoter trap targeted & 2 bp deletion \\
BAZ1B-O4 (-/-) & Promoter trap targeted & 4 bp insertion \\
\hline
\end{tabular}

Table indicates the nature of the mutations generated at the two chromosome $7 B A Z 1 B$ alleles for the clones used in this study. Next to each clone name, $(+/-)$ indicates heterozygous mutant whereas $(-/-)$ indicates homozygous mutant.
297) (Figure 1g). The NHEJ form would contain the WAC and DDT domains only, whereas the double targeted form of the protein would only contain the WAC domain. In order to detect such a protein, we repeated the Western analysis using the second antibody that was raised to the first 50 amino acids of WSTF. Consistent with the first Western, no truncated protein could be detected (Figure 1h).

Loss of WSTF does not impact chromatin of the human Xi At interphase, the $\mathrm{Xi}$ can be readily detected as a densely staining heterochromatin mass, or Barr body, that is typically located in a perinucleolar or peripheral location in the nucleus [41]. The Xi is painted by XIST RNA [14] and facultative heterochromatin markers are readily observed due to the chromosome wide silencing, resulting in an unusually large concentration of these features. Previously, we have shown that WSTF as part of the WICH complex, associates with the Xi territory during S-phase [15] as the chromosome is replicated [42]. In order to determine if WSTF loss impacts the organization and maintenance of $\mathrm{Xi}$ chromatin, we assessed a number of Xi markers for changes in their distribution.

First, we performed RNA FISH to investigate whether the $\mathrm{Xi}$ had retained its association with XIST RNA. Here, we found that XIST RNA was enriched in the vicinity of the Barr body in $B A Z 1 B$ knockouts in a pattern indistinguishable from that of parental RPE1 cells (Figure 2a). Next, we examined other chromatin features of the Xi. Typical characteristics of the human $\mathrm{Xi}$ include an enrichment of H3K9me3 [4,5], H3K27me3 $[7,8]$, and mH2A1 [10], and a noticeable absence of H3K4me2 [4,43] and histone acetylation [44], such as H3K9Ac. By IF, none of these features appeared perturbed in the $B A Z 1 B$ knockouts, having distribution patterns indistinguishable from that observed in parental RPE1 cells (Figure 2b). However, of note, the distribution of H3K9me3 throughout the rest of the nucleus appeared more punctate (Figure 2b, top row). 


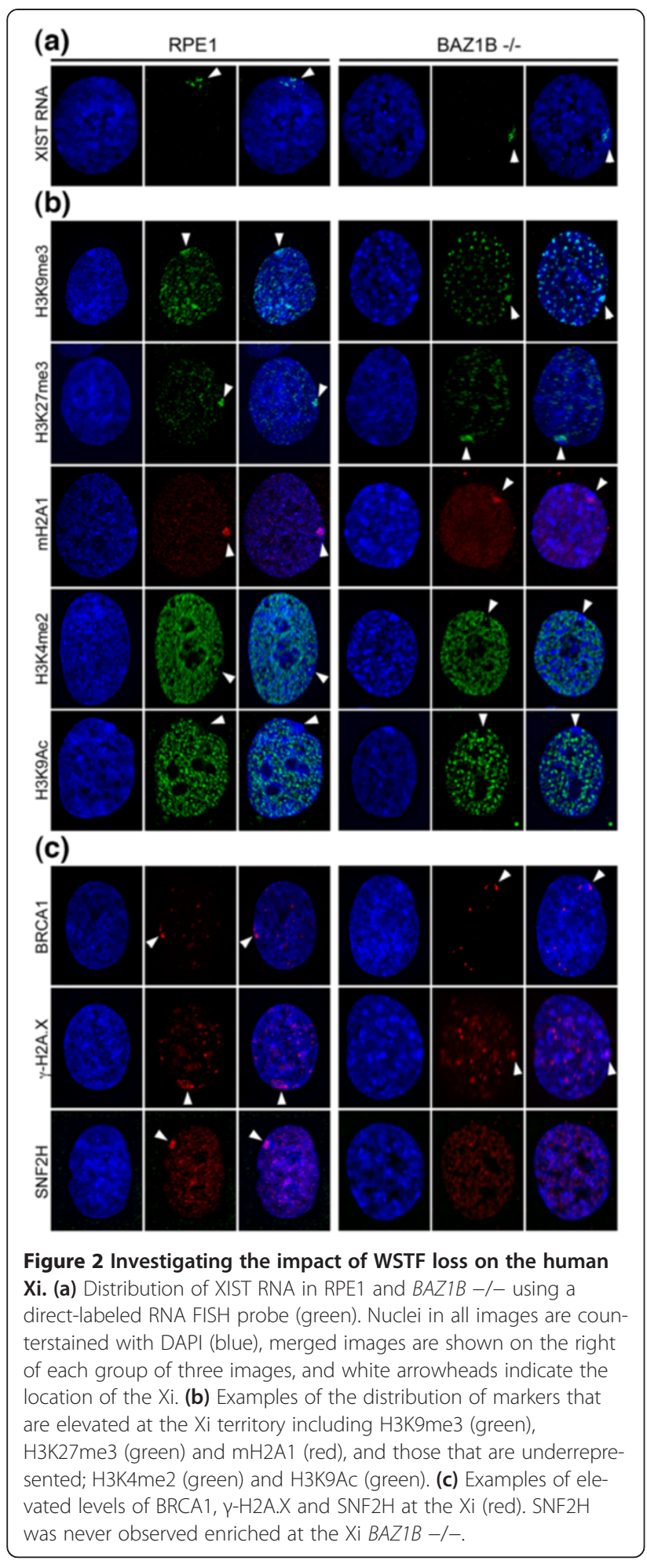

WSTF is one of several proteins that transiently associate with the Xi during S-phase [15]. Other transient features of the $\mathrm{Xi}$ during this time include the association of the breast cancer gene (BRCA1) and the phosphorylation of histone H2A.X at serine 139 ( $\gamma$-H2A.X)
[45]. We sought to determine if these features were altered in the $B A Z 1 B$ knockout cells. Towards this aim, we employed IF using antibodies to BRCA1, $\gamma$-H2A.X, and SNF2H-WSTF's partner in the WICH complex [16]. We found that BRCA1 and $\gamma$-H2A.X still localize to the $\mathrm{Xi}$ $(1.5 \%$ and $2.5 \%$ respectively, $\mathrm{n}=200$ ) (Figure $2 \mathrm{c}$ ), similar to what is reported in the parental cells $[15,45]$. In contrast, SNF2H was no longer detected at elevated levels at the Xi $(n=1242)$. However, SNF2H protein still exhibited diffuse staining at other locations within the nucleus, consistent with its role in other complexes independent of WSTF and WICH [46-49].

\section{Cells lacking WSTF exhibit a unique nuclear phenotype}

DNA staining with 4',6-diamidino-2-phenylindole (DAPI) revealed a nuclear phenotype unique to $B A Z 1 B$ knockout cells. In parental hTERT-RPE1 nuclei, the most intense DAPI staining structure corresponds to the Barr body, as indicated by the white arrow in Figure 3a. In contrast, the nuclei of $B A Z 1 B$ knockout cells show additional DAPI dense bodies of comparable to greater intensity as demonstrated by the pixel saturation in Figure $3 \mathrm{~b}$ and $3 \mathrm{c}$. Not all nuclei show the appearance of these structures. We observed that $23.21 \%$ of nuclei $(n=2356)$ possessed numerous large DAPI-dense regions (Figure 3d). Intriguingly, transient reduction of either component of the WICH complex by RNAi was reported to result in a gain of heterochromatin [18], consistent with these observations.

We sought to further characterize both the composition and dynamics of the DAPI-dense regions, as the appearance of blocks in only a subset of cells raised the possibility that the blocks could be linked to a cell cycle, arrest in proliferation, or potentially early stages of cell death.

\section{DAPI-dense regions are enriched for constitutive heterochromatin markers}

Areas of intense DAPI staining in nuclei typically correspond to regions of heterochromatin, such as the constitutive heterochromatin of mouse major satellite [50], senescence associated heterochromatin blocks [51], or facultative heterochromatin of the $\mathrm{Xi}$ [14]. The blocks seen in the $B A Z 1 B$ homozygous mutants were reminiscent of the heterochromatic Barr body in both DAPI intensity and size. For this reason, we examined the blocks for association with heterochromatic features and various complexes known to interact with heterochromatin. To accomplish this, we pursued several avenues of investigation. First, we explored the relationship of the blocks with the three different HP1 proteins: HP1 $\alpha$, HP1 1 , and HP1 $\gamma$ [52-54]. Chromatin associated HP1 levels were shown previously to increase in cells with depleted levels of WSTF, particularly HP1 $\beta$ [18]. Consistent with this observation, we found that all three HP1 

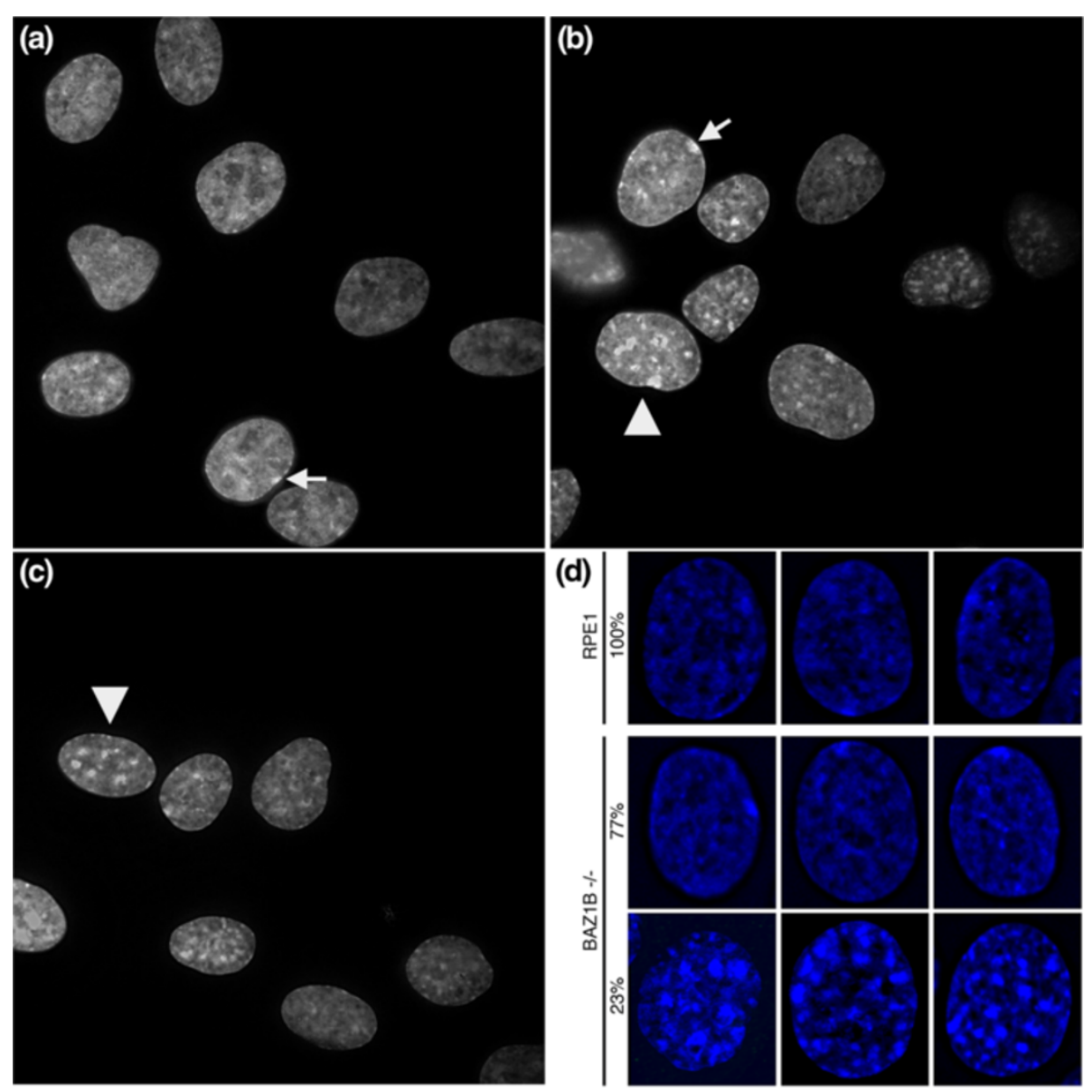

Figure $3 B A Z 1 B$ homozygous knockout cells exhibit a unique nuclear phenotype. Representative images of RPE1 (a), BAZ1B-N6 (b) and BAZ1B-O4 (c) nuclei counterstained with DAPI. White arrow-heads in (b) and (c) point to block-containing nuclei. White arrows point to the DAPIdense Barr body. Images were captured using settings defined for RPE1 that result in pixel saturation for block-containing BAZ1B -/- nuclei. (d) RPE1 and $B A Z 1 B-/$ - nuclei stained with DAPI showing the appearance of large DAPI-dense blocks in a fraction of mutant cells, as indicated to the left.

proteins localize to the DAPI-dense blocks (Figure 4a). To validate that all three isoforms did indeed associate with the blocks and that this was not a by-product of antibody cross-reactivity, we transiently transfected $B A Z 1 B$ knockout cells with $\mathrm{HP} 1 \alpha, \mathrm{HP} 1 \beta$, or $\mathrm{HP} 1 \gamma$ green fluorescent protein (GFP) expression constructs. We found that all three exogenous HP1 types, with HP1 $\alpha$ to a lesser extent, localized to the blocks (Figure 4b).

Next, we continued to characterize the DAPI-dense blocks in the $B A Z 1 B$ knockouts through IF to a variety of heterochromatin-associated proteins and covalent histone modifications. Our results showed that, consistent with binding of HP1 to H3K9me3 [55-57], the blocks were clearly defined by this modification (Figure 4c).

Given the size of the blocks, we wondered if this represented an overall increase in $\mathrm{H} 3 \mathrm{~K} 9 \mathrm{me} 3$ levels. Histones were extracted from parental and WSTF mutant cells and H3K9me3 levels detected by Western blotting. Despite the apparent increase by IF, H3K9me3 levels were comparable between mutants and parental cells
(Figure 4d). However, given that only $23 \%$ of cells show blocks, it is possible that increases are masked by the $77 \%$ of normal cells.

Recently, the structural maintenance of heterochromatin protein, SMCHD1, was linked to HP1 and H3K9me3 [58]. In agreement with this, SMCHD1 associated with the DAPI blocks (Figure 4e). With the exception of the $\mathrm{Xi}$, comparable large blocks of HP1 $\alpha, \mathrm{HP} 1 \beta, \mathrm{HP} 1 \gamma$, H3K9me3 and SMCHD1 were not a feature of parental cells (Additional file 1).

Although they did not completely colocalize, areas of MBD1 and HMG-I/Y enrichment were present within portions of the blocks (Additional file 1). As expected, the blocks had no association with euchromatin markers (Figure 4f). However, 14 other heterochromatin proteins did not associate with the blocks, including TIF1B, EZH2, Mi2, RbAp46, RbAp48, SAP18, SAP30, SIN3A, SIN3B, HDAC1, HDAC2, MBD3, ATRX, and SETDB1, nor histone $\mathrm{H} 1$ or the histone variant macroH2A1 (Additional file 1). 
(a)

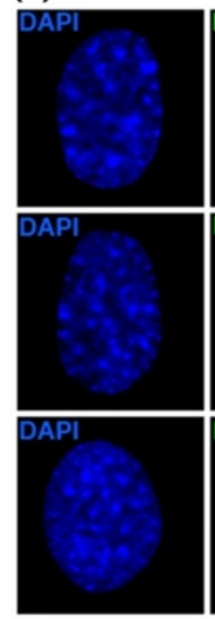

(c)

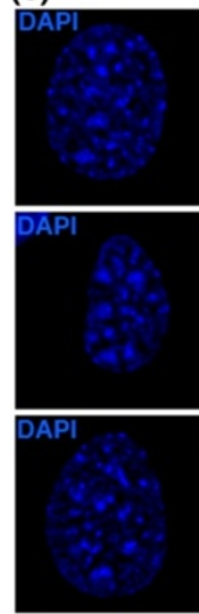

(f)

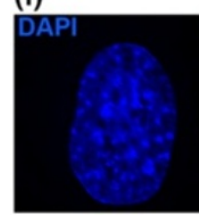

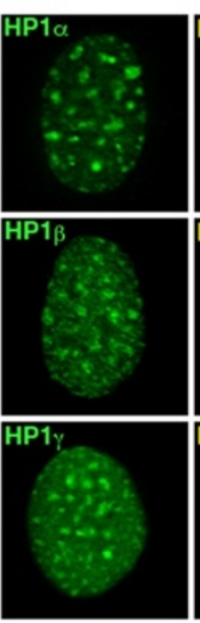
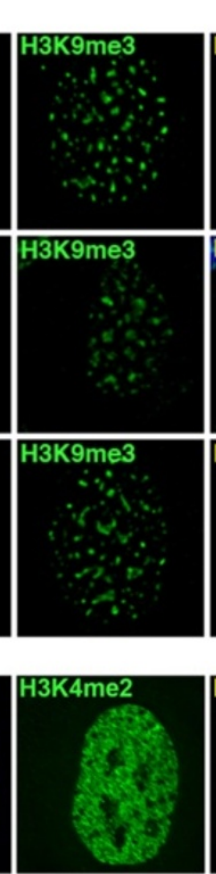
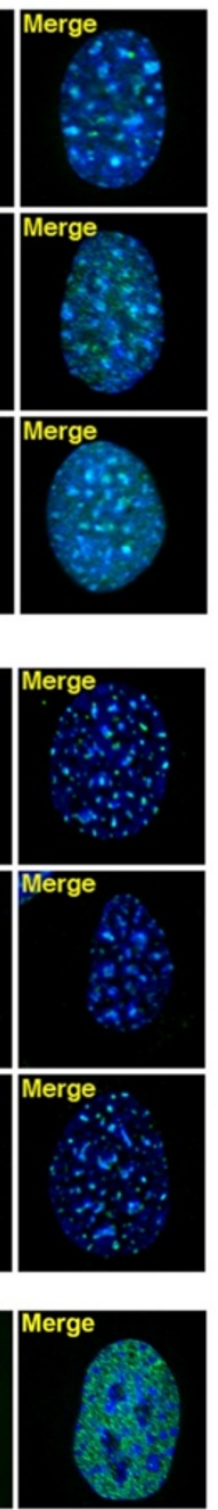

(b)

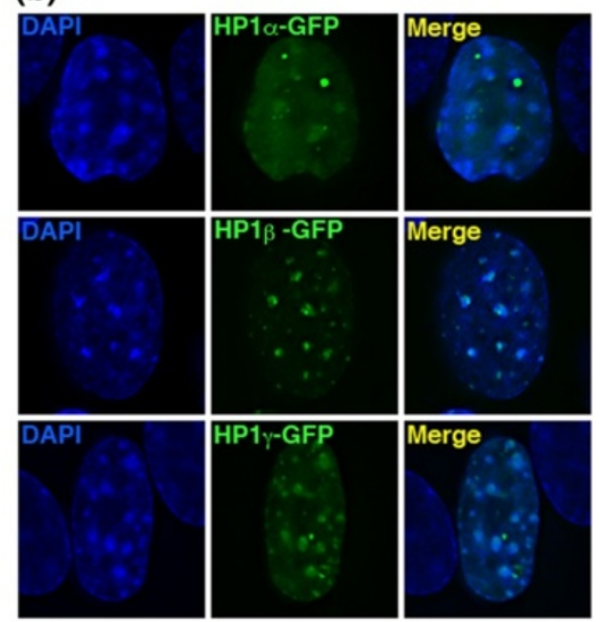

(d)

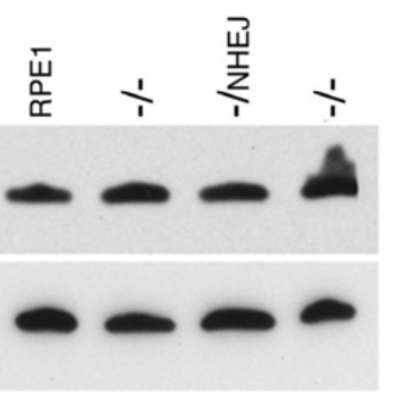

(e)
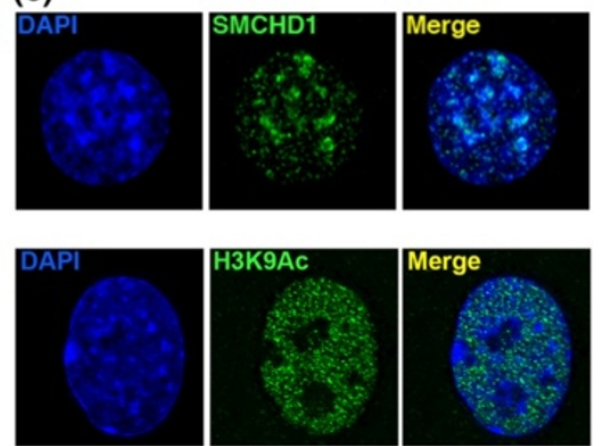

Figure 4 BAZ1B -/- DAPI-dense blocks are characterized by constitutive heterochromatin markers. (a) Association of endogenous HP1a, HP1 1 , and HP1Y (green) with DAPI-dense blocks. Nuclei are counterstained with DAPI (blue) and merged images are shown in the right-side panel. (b) Association of exogenous GFP-tagged HP1a, HP1ß, and HP1Y (green) with DAPI-dense blocks. (c) Examples of elevated levels of H3K9me3 (green) at DAPI-dense blocks. (d) Western blot showing comparable levels of H3K9me3 in mutant compared to RPE1. Anti-histone H4 is used as a loading control. (e) Example of the association of SMCHD1 (green) with DAPI-dense blocks. (f) Examples showing the lack of association of euchromatin markers H3K4me2 and H3K9AC with the DAPI-dense blocks.

DAPI-dense blocks do not correspond to common nuclear structures

We sought to determine if the DAPI-dense regions were associated with disruption of B-WICH activity, due to its participation in ribosomal DNA transcription at nucleoli [20]. The nucleolar organizing region (NOR) of the acrocentric chromosomes $(13,14,15,21$, and 22) localizes to and can be used to define the extent of nucleoli [15]. We performed DNA FISH using a BAC from the NOR as a probe, and IF using an antibody raised against MYBBP1A, a member of the B-WICH complex [20], to examine if the DAPI blocks coincided with nucleoli. We found that neither NOR or MYBBP1A overlapped with the DAPI-dense blocks (Figure 5a).

Given the large number of DAPI-dense blocks, we wondered if they corresponded to the inappropriate spread of centromeric heterochromatin. The reasoning behind this assumption is that cells treated with RNAi 


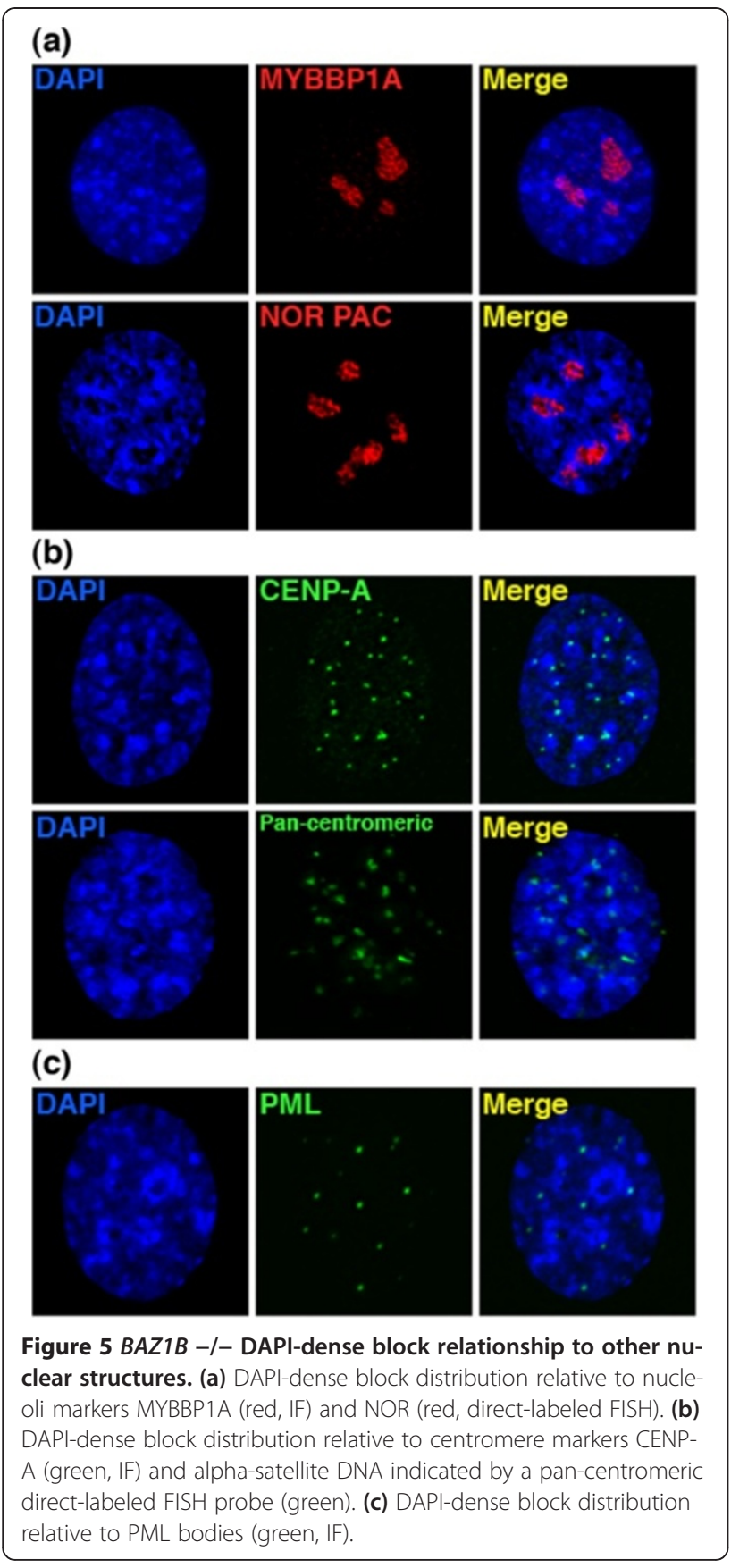

to $B A Z 1 B$ showed an increase in $\mathrm{HP} 1 \beta$, which was postulated to represent gain of heterochromatin [18], and that HP1 is a feature of centromeric heterochromatin [59]. We addressed this two ways, first by DNA FISH with a pan-centromeric probe, and second by IF to the centromeric protein CENP-A $[60,61]$. We found that the blocks did not overlap with either centromeric DNA or CENP-A-positive regions (Figure $5 \mathrm{~b}$ ), although signals often resided at the edge of the blocks.

Prior studies have also linked WSTF function to several cellular processes including DNA replication
(WICH), transcription (B-WICH), and epigenetic silencing (WICH) [62]. Like WSTF, promyelocytic leukemia (PML) bodies have also been linked to the processes of DNA replication, transcription, and epigenetic silencing [63]. In addition, the number, and sometimes size, of PML bodies are reminiscent of the DAPI-dense blocks that we see in $B A Z 1 B$ knockout cells. Altogether, this led us to question whether the DAPI-dense blocks were expanded PML bodies. To test this question, we detected PML bodies by IF. We found that PML bodies had not increased in size and did not colocalize with the DAPI-dense blocks (Figure 5c).

DAPI-dense blocks in $B A Z 1 B$ knockout cells are not linked to and do not disrupt the cell cycle

The appearance of the DAPI-dense blocks in only a subset of cells could be explained by their appearance being associated with a particular period during the cell cycle. In order to test this, we examined cells labeled with markers of the cell cycle: CDT1 for G1 $[64,65]$, PCNA distribution for S-phase [66,67], and phosphorylation of histone $\mathrm{H} 3$ at serine 10 (H3S10ph) for G2 phase [68]. DAPI-dense blocks were detected at all stages of the cell cycle (Figure 6a).

Next, we wondered if the appearance of these blocks affected the progression of the cell cycle. However, cell cycle profiles determined by fluorescence activated cell sorting (FACS) were indistinguishable between parental RPE1 and BAZ1B knockout cells (Figure 6b).

These observations suggest that perhaps cells were slowly acquiring blocks over time, or that only a subset of cells always have blocks and others do not.

\section{Cells containing DAPI-dense blocks do not accumulate over time in culture and are not stably inherited} In order to determine if the number of cells with DAPIdense blocks was accumulating over time, we monitored cells for the percentage of DAPI-dense positive nuclei every 10 passage doublings for a total of 70 doublings. We found that at any given passage doubling, the population of cells with DAPI-dense blocks remained consistently around $20-25 \%$ of cells (Table 2 ).

Since only a subset of cells possessed the DAPI blocks, we questioned whether they were a stable heritable trait that could be passed on to daughter cells. If this were the case, we would expect that clones derived from a non DAPI-dense block containing cell would show no blocks, while those derived from a DAPI-dense block containing cell would all contain blocks. To investigate this question, we isolated single cell clones and examined eight subclones of each of three independent $B A Z 1 B$ homozygous knockout mutants for the presence or absence of the DAPI-dense blocks. Interestingly, all subclones contained cells with and without DAPI-dense 


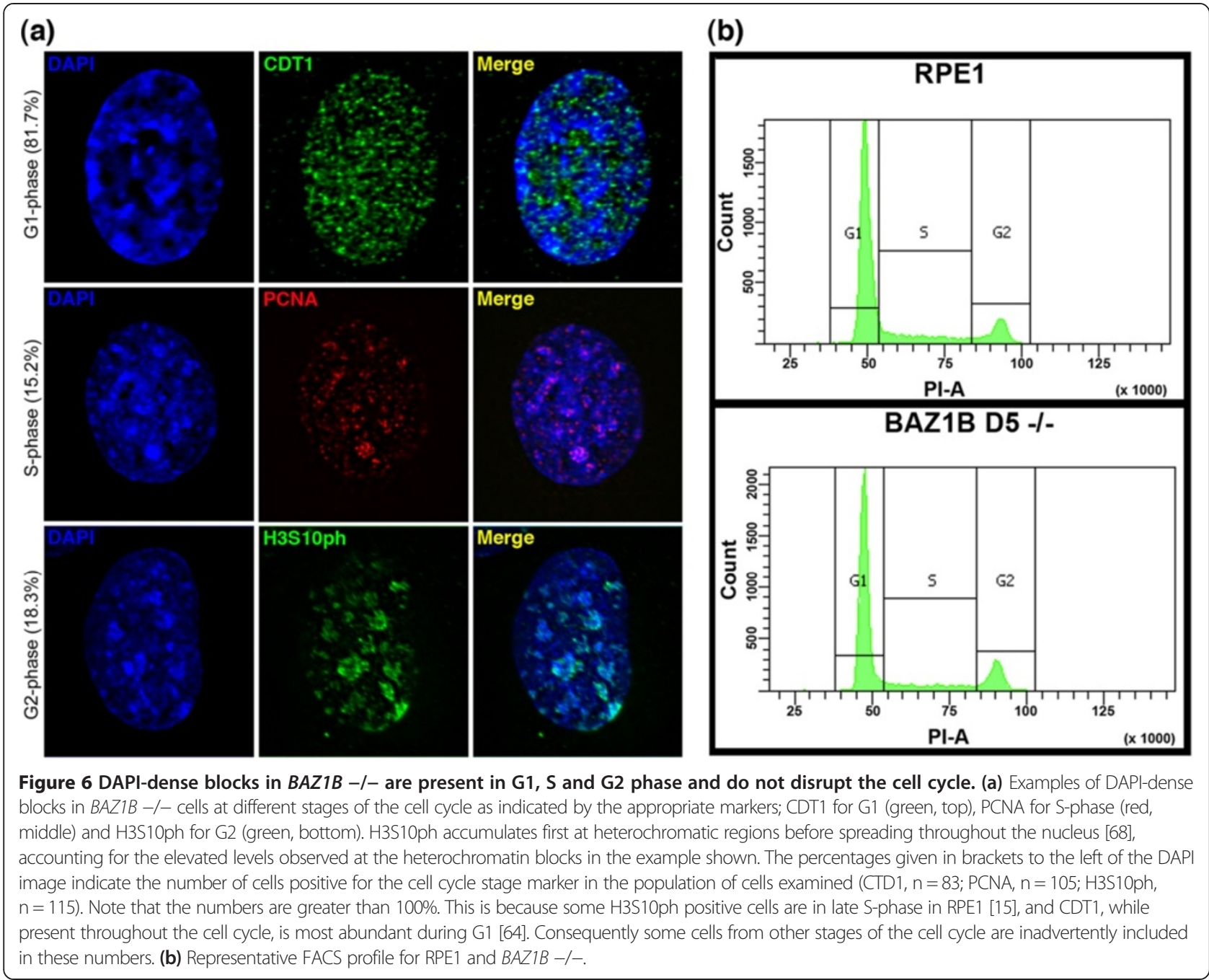

Table 2 Appearance of DAPI-dense blocks does not change with passage doubling

\begin{tabular}{ccc}
\hline $\begin{array}{c}\text { Passage } \\
\text { doubling }\end{array}$ & $\begin{array}{c}\text { Percentage of nuclei } \\
\text { with DAPI-dense blocks }\end{array}$ & $\begin{array}{c}\text { Number of } \\
\text { nuclei scored }\end{array}$ \\
\hline 14 & 31.85 & 135 \\
24 & 22.74 & 277 \\
34 & 22.94 & 327 \\
44 & 20.27 & 291 \\
54 & 21.30 & 315 \\
64 & 24.46 & 372 \\
74 & 22.14 & 262 \\
84 & 24.13 & 377 \\
\hline
\end{tabular}

Table shows the percentage of BAZ1B -/- clone D5 nuclei that showed DAPIdense blocks over the course of 70 passage doublings. A total of 2356 nuclei were scored. The mean and standard deviation is $23.73 \%+/-3.56$. blocks (Table 3), which suggested that cells could develop and resolve blocks regardless of the state of the parent cell. However, it remains a possibility that those cells acquiring blocks arrest in the cell cycle and eventually die.

\section{DAPI-dense blocks are dynamic}

The transient transfection of HP1 $\beta$ into $B A Z 1 B$ knockout cells clearly highlighted the DAPI-dense blocks (Figure $4 \mathrm{~b}$ ),

Table 3 DAPI-dense block incidence in single cell clones

\begin{tabular}{lcc}
\hline $\begin{array}{l}\text { BAZ1B } \\
-/- \\
\text { clone }\end{array}$ & $\begin{array}{c}\text { Percentage of nuclei with DAPI-dense } \\
\text { blocks for } 8 \text { independent subclones }\end{array}$ & $\begin{array}{c}\text { Mean \& standard } \\
\text { deviation }\end{array}$ \\
\hline D5 & $78,50,76,78,44,58,40,48$ & $59.0 \%+/-16.0$ \\
F3 & $8,40,22,56,24,42,20,24$ & $29.5 \%+/-14.0$ \\
M1 & $20,28,20,18,22,20,32,38$ & $24.8 \%+/-7.0$ \\
\hline
\end{tabular}

Table shows the percentage of nuclei with DAPI-dense blocks for each of eight single-cell subclones derived from three independent BAZ1B -/- clones. Fifty nuclei were scored for each subclone. 
providing an opportunity to monitor live cells expressing the GFP construct in real time. Cells expressing HP1 $\beta$ GFP were followed over 24 hour and 72 hour periods. Since the number of block-containing cells did not increase in the population over time, the blocks could either be spontaneously forming and resolving, or those cells with blocks could arrest, or undergo apoptosis. We observed that the block-containing nuclei did not undergo apoptosis, but were readily able to divide, ruling out arrest in the cell cycle (See Additional file 2). We found that a block-containing cell was able to give rise to a daughter cell that also acquired blocks, and, therefore, the condition was mitotically heritable (Additional file 3). Furthermore, the blocks appear to resolve with the exception of the $\mathrm{Xi}$ associated HP1 $\beta$-GFP block, highlighting the dynamic nature of the blocks. Interestingly, we noted that, despite substantial movement by the cell body, the blocks were static within nuclei (Additional file 4), similar to observations made in real-time with replication factories [67]. Finally, blocks were observed for as long as 24 hours in cells before dividing, and appeared to resolve right before entering mitosis, confirming that they are not transient features of a particular cell cycle stage (Additional file 3). Given these observations and the fact that single cell clones give rise to a similar fraction of cells with DAPI-dense blocks as the parental cell population (and not 100\% blockcontaining or $100 \%$ block-free), we conclude that the appearance and resolving of the blocks is a dynamic process. What triggers the formation and subsequent resolution of the blocks in some cells and not others is presently unknown.

\section{Introduction of exogenous full length WSTF ORF is} sufficient to remove the appearance of DAPI-dense blocks We wondered if the DAPI-dense blocks in $B A Z 1 B$ knockout cells could be removed by reintroducing wild type WSTF protein. To test this, an expression construct containing a Myc-tagged full-length WSTF ORF was introduced into WSTF knockout cells by Nucleofection. Cells were examined by IF using antibodies specific to the Myc-tag and WSTF. Cells that were positive for Myc were also positive for WSTF $(n=94)$ (Figure 7a). Furthermore, transfected cells were restored for transient association of WSTF to the $\mathrm{Xi}$ as defined by H3K27me3 [15] (Figure 7b). We examined Myc-positive nuclei for DAPI-dense blocks. All Myc-positive nuclei showed DAPI patterns comparable to parental RPE1, with no evidence of intense DAPI-dense blocks $(n=119)$, suggesting that the blocks can be resolved by reintroduction of wild-type protein.

\section{Loss of WSTF results in gene expression changes}

Given that the DAPI-dense blocks are enriched for markers of constitutive heterochromatin, and that WSTF

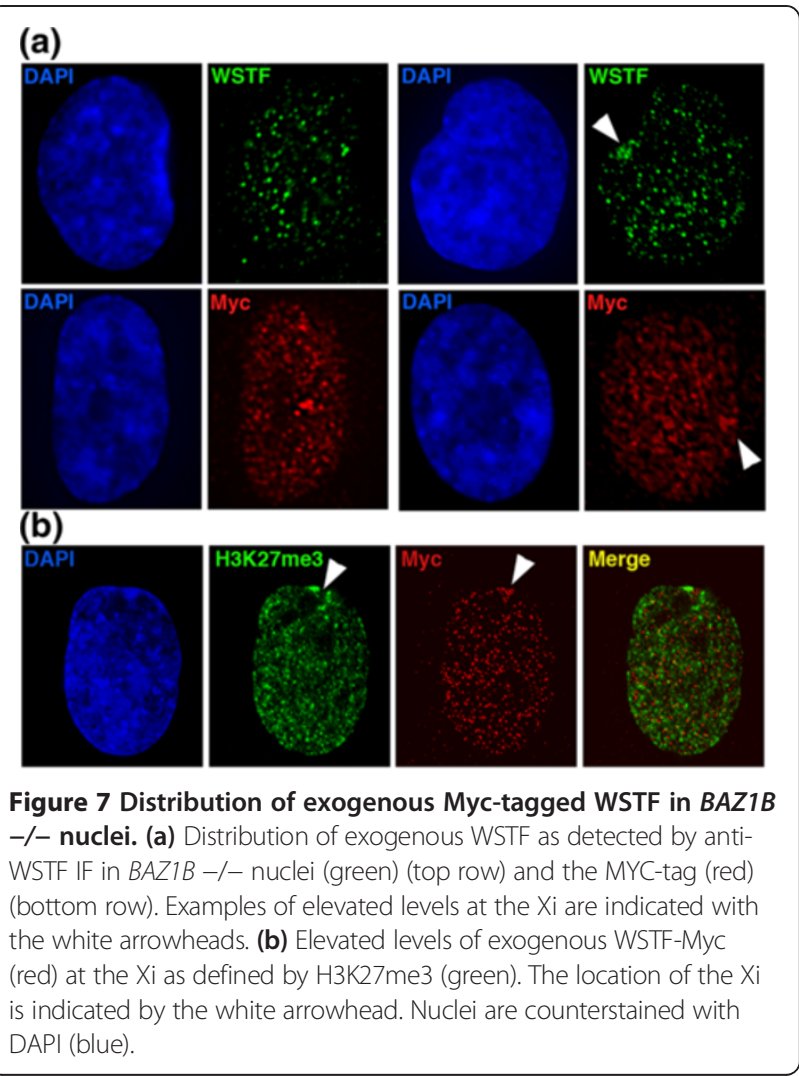

is involved in transcription [62], we assessed the WSTF mutant cells for changes in gene expression, with the thought that perhaps most gene expression changes would be reductions due to the gain in heterochromatin. Total RNA was extracted from three separate cultures of parental RPE1 cells as well as from three separate cultures for each of three independent $B A Z 1 B$ knockout clones, and one heterozygous mutant clone. Residual DNA in the RNA preparation was digested and its removal confirmed by PCR before preparing cDNA, labeling and hybridizing to a NimbleGen human gene expression $12 x 135 \mathrm{~K}$ array containing $>45,000$ transcript targets. Genes that had significant changes of two fold or more compared to RPE1 ( $p<0.05$ ) were identified for each mutant. The heat map shown in Figure 8a, clearly shows consistent and substantial changes in gene expression between the three independent $B A Z 1 B$ mutants as compared to the parental RPE1 cells. The genes that were not shared between all three independent mutants were excluded. The remaining genes of interest can be found in Additional file $5(n=515)$. In the knockout cells, genes for which expression levels changed were not all reductions; some went up (173) while others went down (342). To validate the microarray data, we performed quantitative RT-PCR (qRT-PCR) on several genes that showed increases or decreases in the microarray data relative to the parental RPE1 cells. All 


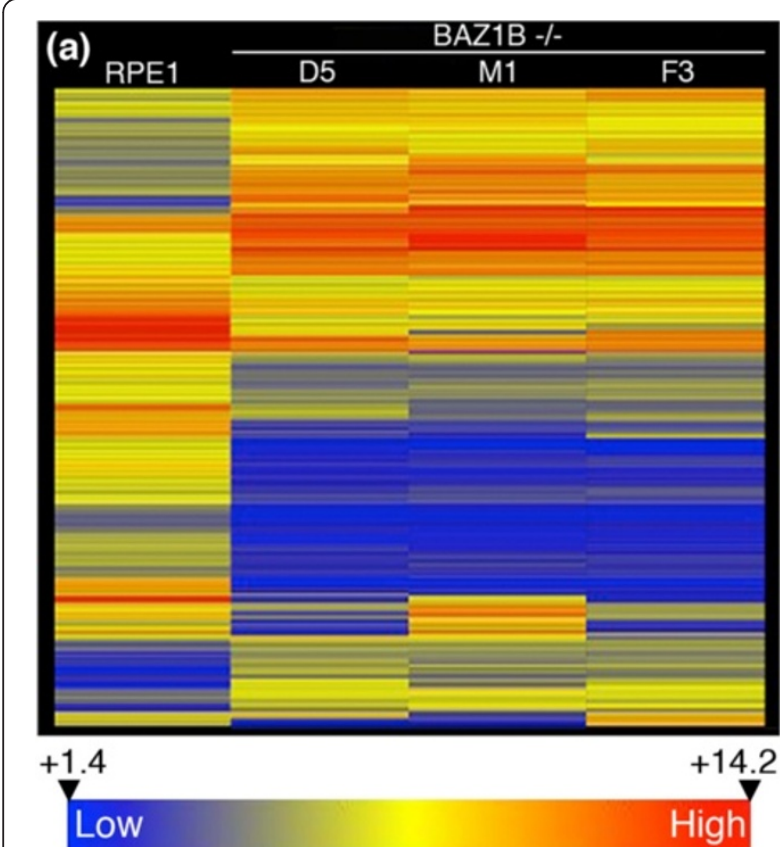

(b)

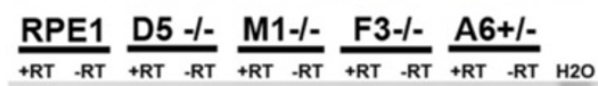

GAPDH
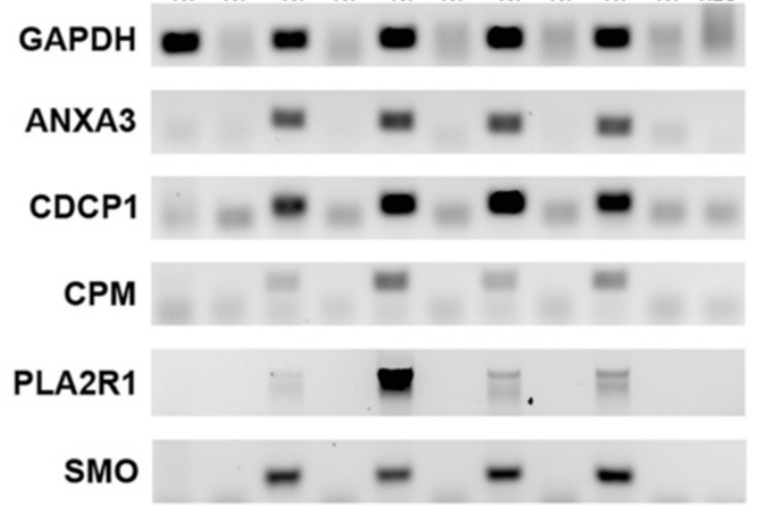

Figure 8 Gene expression changes in $B A Z 1 B-/-$ cells. (a) Dendrogram (Heat Map) of hierarchical clustering of gene expression between RPE1 and three independent BAZ1B -/- clones. Heat map represents 586 data points, corresponding to probes showing greater than 2-fold change in gene expression at 95\% confidence. Expression levels range from +1.4 to +14.2 , corresponding to signal intensity on the microarray and represented by a blue (low) to red (high) gradation indicated by the scale bar beneath the heat map. Each column represents the averaged signal from three replicate data sets for each clone. Each row represents a single specific probe. Changes in gene expression can be seen by the difference in row colors between the mutants and parental cells. (b) Representative examples of genes activated in BAZ1B -/cells by standard end-point RT-PCR analysis shown as an inverted image of an ethidium bromide stained agarose gel. The gene name is given to the left, and the cell line indicated at the top. Lanes show with (+RT) and without $(-\mathrm{RT})$ reverse transcriptase and a water control $(\mathrm{H} 2 \mathrm{O})$.

consistently showed the same trend (data not shown). Interestingly, of the 515 gene expression changes observed in the $B A Z 1 B$ knockouts, 72 of these were shared in the heterozygous mutant cells (Additional file 6). This indicates a degree of sensitivity to WSTF haploinsufficiency, further supporting an important contributing role of $B A Z 1 B$ hemizygosity in WBS.

Despite our supposition that most genes would be silenced due to the gain in heterochromatin, as mentioned above, a substantial number of genes showed increased expression in the mutants relative to the parental cells. Among these, a subset appeared to show activation in both $B A Z 1 B-/-$ and $B A Z 1 B+/-$ cells (Figure $8 b$ ). These genes could not be assessed by qRT-PCR, as fold change cannot be determined when expression of a control or unknown sample is zero, but the endpoint RTPCR analysis is consistent with the microarray data.

Gene expression changes were not restricted to certain chromosomes or chromosomal regions (Additional file 5). With the exception of chromosome 21, all chromosomes have genes that showed increases or decreases in expression, although all changes were less than $2.0 \%$ of the total gene content for the chromosome (coding and non-coding). Given that chromosome 21 has the lowest gene content, it does not seem that this chromosome is an exception for any biological reason. Despite the blocks frequently residing close to nucleoli, the NORcontaining chromosomes (13, 14, 15, 21 and 22) did not have the highest number of silenced genes. Instead, the chromosomes that contained the largest number of silenced genes were chromosomes $20(1.55 \%)$ and 12 (1.50\%). No obvious trend was observed for silenced genes concerning genomic location, such as residing close to large satellite blocks or in pericentromeric or subtelomeric regions. Interestingly, the protocadherin cluster on chromosome $5 \mathrm{q} 31.3$ that is associated with various neuropsychiatric disorders [69], and implicated in tumor suppressor activity [70,71], consistently showed an increase in expression of most of the alpha members, but decreases in the adjacent beta and gamma clusters. Intriguingly, in the heterozygous $B A Z 1 B$ mutant, the alpha cluster was up-regulated, similar to the complete knockout, but changes to the beta and gamma clusters were not observed.

\section{Discussion}

Gene silencing at the $\mathrm{Xi}$ is remarkably stable, involving multiple redundant gene silencing mechanisms that ensure silencing is maintained throughout all subsequent somatic cell divisions. Stability of the imprinted $\mathrm{Xi}$ in extraembryonic material is compromised upon loss of the Polycomb group protein Embryonic Ectoderm Development (EED) $[72,73]$, but there are few other examples of $\mathrm{Xi}$ instability due to a single gene loss. In fact, global reactivation of the Xi has only been achieved through fusion of female mammalian cells with mouse embryonal carcinoma cells [74-77] or via reprogramming mouse female cells to pluripotency through forced expression of 
transcription factors [78]. Therefore, if WSTF is involved in maintaining chromatin states at the $\mathrm{Xi}$, it is not surprising that the loss of WSTF did not result in an obvious wave of X-linked gene reactivation and loss of bonafide Xi markers, such as XIST or H3K27me3. Genes that are reactivated from the $\mathrm{Xi}$ are unlikely to be expressed at levels dramatically different from their Xa counterpart. Consequently, the anticipated change in gene expression would likely be less than two fold. However, reassessing the data for 1.5 fold change did not show a bias toward increase in X-linked gene expression levels compared to the autosomes (data not shown). Alternatively, however, these observations can be interpreted to suggest that perhaps the transient association of WSTF with the chromosome, as the Xi DNA is replicated [15], is unrelated to maintenance of XCI.

WSTF, as part of the WICH complex, has been shown to transiently associate with the Xi prior to $\gamma$-H2A.X and BRCA1 [15]. The fact that BRCA1 and $\gamma-\mathrm{H} 2 \mathrm{~A} . \mathrm{X}$ continue to transiently associate with the Xi when WSTF is absent suggests that the two cell-cycle linked events are not coupled. Indeed, we have previously speculated that WICH may transiently associate with the newly replicated DNA in order to reestablish the basal state of H2A.XY142ph. This theory supports the idea that WSTF recruitment to the $\mathrm{Xi}$ is independent of $\gamma-\mathrm{H} 2 \mathrm{~A} . \mathrm{X}$ and BRCA1 association since the ground state of H2A.X-Y142ph is set independently of DNA damage [21].

Intriguingly, almost one quarter of $B A Z 1 B$ knockout cells displayed numerous, large, DAPI-dense blocks. Consistent with the DAPI staining, the blocks are characterized by constitutive heterochromatin-associated proteins, including HP1, HMG-I/Y, and MBD1, and by the covalent histone modification H3K9me3. In addition, the HP1interacting protein SMCHD1 [58], was also seen localizing to the blocks. While the blocks did not overlap with nucleoli, they were often seen abutting these nuclear structures, as indicated by NOR DNA FISH and MYBBP1A IF; markers of nucleoli [15]. Prior studies have shown that, in addition to rDNA-containing regions of the acrocentric chromosomes, heterochromatin domains of most human chromosomes (including regions found at telomeres, centromeres, and chromosome arms), associated with nucleoli [79]. Perhaps, perinucleolar regions are sites of heterochromatin maintenance. Indeed, in mouse, the binding partner of Wstf, Snf2h, transiently accumulates in a perinucleolar region as the mouse $\mathrm{Xi}$ is replicated [80]. Perhaps, in BAZ1B knockout cells, checks on heterochromatin formation are compromised resulting in the inappropriate spread into genomic regions not normally associated with heterochromatin. The obvious gain in the large blocks of heterochromatin support a model in which WSTF assists in maintaining chromatin states, and in its absence, too much heterochromatin forms [17].
The size and number of DAPI-dense blocks in $B A Z 1 B$ knockout cells indicates that the gain of heterochromatin in affected cells is substantial. Previously, it was shown that artificially reducing WSTF protein levels by RNA interference, was associated with lower levels of transcription, as measured by monitoring the levels of bromouridine incorporation into RNA [18]. By microarray analysis, a large number of genes consistently showed a reduction in expression (342) in three independent mutants. This change could be attributed to the gain in heterochromatin, although secondary effects, such as silencing of a key transcription factor, cannot be ruled out. Of interest, the reduced expression was not global, impacting only a subset of all genes. This suggests that if WSTF is acting to reestablish appropriate chromatin states post DNA replication, as postulated [17], it is either only acting at specific regions of the genome, as most genes are unaffected by the proteins loss, or that certain regions of the genome are more vulnerable to inappropriate heterochromatin spread/assembly than others.

We also observed a large number of genes that showed an increase in expression (173) in all three $B A Z 1 B$ knockout clones. This is consistent with WSTF's previously established role as a regulator of transcription, capable of activating or silencing genes [62]. Notably, loss of a single $B A Z 1 B$ allele was sufficient to impact a subset of those genes affected in the knockouts, indicating that some genes are more sensitive to WSTF levels than others. This has important implications for $B A Z 1 B$ hemizygosity in WBS, and our heterozygous targeted clones provide an important model in which to pursue the contribution of WSTF to the phenotype in this complex contiguous gene deletion disorder.

Some genes showed reactivation from a silent state in the mutants. In contrast, relatively few genes showed the opposite trend of complete silencing in the mutants. It is possible that within the pool of cells assessed, complete silencing might only be observed in cells with DAPIdense blocks that represent fewer than $25 \%$ of the total cell population. In these cells, genes embedded in the blocks or within their vicinity might be fully silenced; yet not in the $75 \%$ of cells that appear normal. Continuing with this notion, it is possible that the actual number of genes impacted by WSTF loss might be greater than our microarray data would otherwise suggest. If some genes show increases or decreases in gene expression that are close to, but greater than, our 2-fold cutoff only in DAPI-dense block nuclei, these changes might be reduced to below the cutoff by a lack of change in the majority non-DAPI-dense block cells.

Despite the extensive size of the DAPI blocks, we did not see an increase in nuclear levels of H3K9me3. Therefore, an alternative explanation for the reactivation 
of genes could be an indirect effect, as H3K9me3 is redistributed to the blocks, essentially reducing local repressive effects elsewhere.

The dynamic nature of the blocks is intriguing. Cells that acquire blocks do not undergo apoptosis and the cell cycle is apparently unaffected. Single cell cloning indicates that block formation is reversible, and that the overall number of cells with blocks remains relatively constant within an actively dividing population and between independent mutants. Live cell imaging confirms that block appearance is not linked to the cell cycle, and indicates that blocks are essentially immobile, and do not prevent cells from proceeding through mitosis. Reintroduction of wild-type WSTF resolves the blocks, and therefore provides a powerful manipulable system in which to investigate regions of the WSTF protein that are required for this activity.

\section{Conclusions}

In this study, we have explored the impact of WSTF loss. Our findings emphasize that many aspects of the function of WSTF remain enigmatic. Identifying the common characteristics of the DNA sequences in the DAPI-dense blocks may provide clues as to how WSTF contributes to gene regulation. It is important for future studies to examine the mechanism of WSTF action so that we may fully understand the role that WSTF plays in nuclear processes and its contribution to WBS. In addition, our heterozygous mutant cells provide a useful model to investigate the effects of $B A Z 1 B$ hemizgosity and WSTF haploinsufficiency in the absence of the loss of the other 27 genes seen in WBS.

\section{Methods}

\section{Cell culture}

The cell line used to generate the knockout clones was hTERT-RPE1, a 46, XX telomerase-immortalized human retinal pigment epithelial cell line (Clontech, Laboratories, Inc., Mountain View, CA, USA: No. C4000-1). The cells were maintained according to the supplier's recommendations.

\section{Generating $B A Z 1 B$ knockout cell lines}

The targeting template for homology mediated repair was generated as follows. The left homology arm (HA) was amplified by PCR with the primers LHA-F2 and LHA-R2 (Additional file 7) using RPE1 genomic DNA as a template. The same was done for the right HA with the primers RHA-F1 and RHA-R1 (Additional file 7). The PCR products for the left and right HAs were TA cloned into pDRIVE (Qiagen, Valencia, CA, USA). Plasmid DNA was harvested using the Nucleospin Plasmid kit (Machery-Nagel, Bethlehem, PA, USA), before sequence verifying. The insert of the left HA was excised from the pDRIVE vector using NotI and XbaI, and the right HA was excised using XhoI and MluI. The pSEPT cassette was digested with $\mathrm{XbaI}$ and $\mathrm{XhoI}$ and the $\mathrm{pBC}$ KO-tk-A vector (Chadwick, manuscript in preparation) was digested with NotI and MluI. All digests were separated on agarose gels (Agarose Unlimited, FL, USA), and inserts excised and purified using a Nucleospin Gel and PCR Clean-up kit (Machery-Nagel, Bethlehem, PA, USA). The left HA, pSEPT cassette, and right HA, were ligated overnight at $16^{\circ} \mathrm{C}$ into $\mathrm{pBC}-\mathrm{KO}$-tk-A vector using T4 DNA ligase (NEB, Ipswich, MA, USA). The ligation was transformed into NEB-5alpha chemically competent E. coli cells according to the supplier's recommendations (NEB, Ipswich, MA, USA). Plasmid DNA was prepared using the Plasmid Plus Midi kit (Qiagen, Valencia, CA, USA). The plasmid DNA was linearized by digestion with $A s c$ I restriction enzyme, and gel purified. To generate $B A Z 1 B$ knockout cell lines, we introduced ZFN expression constructs (Sigma-Aldrich, St. Louis, MO, USA: Cat No. CSTZFN-1KT) and the linearized repair template by Nucleofection (Lonza Group Ltd, Basel, Switzerland). Twenty-four hours post-Nucleofection, cells were seeded into four 96-well plates in media supplemented with neomycin $(150 \mu \mathrm{g} / \mathrm{mL})$. After approximately two weeks, clones were expanded and genomic DNA isolated using a Wizard SV 96 Genomic DNA Purification System kit (Promega, Madison, WI, USA). Correctly targeted clones were screened on the left and right sides by PCR between a primer to the genomic locus, but distal/proximal to the HA, and a primer located in the pSEPT cassette. The screening pairs were Left-Screen-F2 with pSEPT-R1 and pSEPT F2 with Right-Screen-R1 (Additional file 7). Clones that generated the correct sized PCR product using these two screening pairs were tested for NHEJ by PCR using the primers $B A Z 1 B$ Sigma-F and $B A Z 1 B$ Sigma-R (Additional file 7). If a product was generated, it was sequenced to determine if NHEJ had resulted in insertions or deletions. All restriction enzymes were obtained from NEB (Ipswich, MA, USA).

\section{WSTF rescue}

A full-length wild-type human myc-tagged WSTF expression construct was obtained from Origene (Rockville, MD, USA: Cat.No. RC216159). The expression construct was introduced into $B A Z 1 B$ knockout cells by Nucleofection using a 4D-Nucleofector (Lonza Group Ltd, Basel, Switzerland) and solution SF on program EN-138 according to the manufacturers recommendations.

\section{Antibodies}

\section{Immunofluorescence}

Rabbit anti-WSTF antibodies were obtained from Cell Signaling Technology (Danvers, MA, USA: Cat No. 2152) and Bethyl Laboratories (Montgomery, TX, USA: Cat No. A300-446A). Rabbit anti-SMCHD1 (Cat No. 
A302-872A) was also purchased from Bethyl Laboratories (Montgomery, TX, USA). Rabbit anti-CDT1 (Cat No. 10/2012), rabbit anti-SETDB1 (Cat No. 06/2012), rabbit anti-CENP-A (Cat No. 2186S), mouse anti-TIF1B (Cat No. 5868S), and mouse monoclonal antibodies to myc (Cat No. 2276S) were purchased from Cell Signaling Technologies (Danvers, MA, USA). Mouse monoclonal antibodies BRCA1 (Cat No. sc-6954) were obtained from Santa Cruz Biotech (Dallas, Texas, USA) as were goat anti-SAP18 (Cat No. sc-8473), mouse anti-Histone H1 (sc-8030), goat anti-MBD1 (Cat No. sc-9395), goat antiMBD2 (Cat No. sc-12444), goat anti-MBD3 (Cat No. sc-9402), goat anti-RbAp46 (Cat No. sc-8272), goat antiRbAp48 (Cat No. sc-8270), goat anti-ATRX (Cat No. sc-10080), rabbit anti-SIN3A (Cat No. sc-994), rabbit anti-SIN3B (Cat No. sc-768), rabbit anti-Mi2 (Cat No. sc-1564), and rabbit anti-HMG-I/Y (Cat No. sc-1564). Antibodies to Mouse monoclonal antibodies to phospho-H2A.X (Serine-139) (Cat No. 05-636) and SNF2H (Cat No. 05-698) were obtained from Millipore (Billerica, MA, USA), as were rabbit polyclonal antibodies to EZH2 (Cat No. 07-400), histone H3 trimethylated at lysine-27 (Cat No. 07-449), histone H3 phosphorylated at serine 10 (Cat No. 06-570), histone H3 dimethylated at lysine 4 (Cat No. 07-0030), histone H3 acetylated at lysine 9 (Cat No. 06-942), and histone H3 trimethylated at lysine 9 (Cat No. 07-523). Mouse anti-HP1 $\alpha$ (Cat No. MAB3584), anti-HP1 $\beta$ (Cat No.

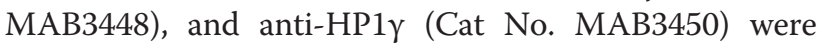
obtained from Chemicon International (EMD Millipore, Billerica, MA, USA). Mouse anti-PML (Cat No. ab96051) antibody was purchased from Abcam (Cambridge, MA, USA). Mouse anti-MYBBP1A (Cat No. SAB1400390) was obtained from SIGMA-Aldrich (St. Louis, MO, USA). Rabbit anti-macroH2A.1 antibody was described previously [81]. Alexa-Fluor ${ }^{\circledR}$ conjugated secondary antibodies were obtained from Life Technologies Corporation (Invitrogen, Grand Island, NY, USA).

\section{DNA and RNA FISH probes}

Direct-labeled FISH probes were prepared using a Nick Translation kit with SpectrumGreen dUTP according to the manufacturer's recommendations (Abbott Molecular, Abbott Park, IL, USA). P1-artificial chromosome (PAC) RP5-1174A5, which contains rDNA and detects the Nucleolar Organizing Regions (NOR), was obtained from Dr. P. Finelli (Finelli et al. 2012). The pan-centromeric probe (Cat No. 1695-F-02) was purchased from Cambio (Cambridge, UK). The XIST RNA-FISH probe was as described previously [15].

\section{Western blotting}

The protein samples for Western blotting were prepared by lysing approximately one million cells in RIPA buffer according to standard procedures [82]. Samples were separated on 4-20\% acrylamide gels at $200 \mathrm{~V}$. Histone samples for Western blotting were prepared according to Abcam's histone extraction protocol (http://www.abcam. com/index.html?pageconfig=resource\&rid=11410). Samples were separated on AnykD acrylamide gels (Biorad, Hercules, CA, USA) at $200 \mathrm{~V}$. All SDS-PAGE gels were transferred to pre-equilibrated $0.2 \mu$ PVDF (Biorad, Hercules, CA, USA) using a BioRad Turbo Transfer apparatus. The PVDF membranes were blocked with 5\% non-fat dry milk in $0.1 \%$ PBS-Tween 20 for 30 minutes and washed twice in $0.1 \%$ PBS-Tween $20 / 1 \%$ igepal for 15 minutes each. Primary antibodies were diluted in blocking solution and either incubated with gentle rocking overnight at 4C (WSTF Cell Signaling Technologies and Bethyl Laboratories antibodies) or for 1 hour at room temperature (all other antibodies). The membranes were washed twice as described above. The membranes were incubated with horseradish peroxidase-conjugated (HRP) secondary antibodies diluted in blocking solution for 1 hour at room temperature with gentle rocking. The membranes were washed as previously described. Detection of HRP was achieved by incubating membranes with equal volumes of ECL Solution A and Solution B (GE Healthcare, Pittsburgh, PA, USA) for 3 minutes at room temperature. The two WSTF antibodies described above were used. Rabbit anti-B-Actin (Cat No. 11/2012) was purchased from Cell Signaling Technology (Danvers, MA, USA). The rabbit anti-H3K9me3 antibody described above for IF analysis was used for Western analysis. Rabbit antiHistone 4 (Cat No. 07-108) was obtained from Millipore (Billerica, MA, USA). HRP anti-rabbit secondary antibody was obtained from Cell Signaling Technology (Danvers, MA, USA: Cat No. 7074S).

\section{DNA and RNA FISH and indirect immunofluorescence}

To prepare slides for IF and FISH studies, approximately $2 \times 10^{5}$ cells were grown directly on standard microscope slides. IF was performed as described [15]. DNA was counterstained using ProLong ${ }^{\odot}$ Gold antifade reagent supplemented with 4',6-diamidino-2-phenylindole (DAPI) (Invitrogen, Grand Island, NY, USA). For IF combined with DNA FISH, IF was first performed. The FISH probes were denatured in the presence of human Cot-1 DNA (Invitrogen, Grand Island, NY, USA) at $78^{\circ} \mathrm{C}$ for 10 minutes followed by 30 minutes at $37^{\circ} \mathrm{C}$ to block repetitive sequences before applying to the slides. Slides were then treated as previously described [15]. Antifade containing DAPI was added to the slides, followed by a cover slip. IF combined with RNA FISH was performed essentially as described [83].

\section{Image acquisition}

Images were collected using a DeltaVision pDV (GE Healthcare, Pittsburgh, PA, USA). Delta Vision images 
were deconvolved with softWoRx 3.7.0 (Applied Precision, Issaquah, WA, USA) and compiled with Adobe Photoshop CS2 (Adobe Systems, San Jose, CA, USA). Live cell imaging videos were recorded using an Andor Revolution Spinning Disk Laser Confocal microscope and an Andor 897 EMCCD camera (Andor Technology plc. Belfast, UK). Live cell imaging data was processed using Andor iQ2 software.

\section{HP1-GFP Expression constructs}

GFP-tagged HP1- $\alpha, \beta$ and $\gamma$ expression constructs were generated by excision with EcoRI of the full ORF inserts from previously described pcDNA3.1-CT-Myc constructs [84], into the EcoRI site of pcDNA3.1-CT-GFP (Invitrogen, Grand Island, NY, USA).

\section{Fluorescence activated cell sorting (FACS) analysis}

For FACS analysis, an asynchronously growing population of $1 \times 10^{6}$ cells were stained with propidium iodide [85] and flow sorted using a FACSCanto (BD Bioscience, San Jose, CA, USA). Cell cycle profiles were generated using FACSCanto software.

\section{Microarray gene expression analysis}

Microarray expression analysis was performed on parental cells as well as three independent knockout mutants and one heterozygously targeted clone. For each sample, total RNA was extracted from three independent cell pellets isolated at different time points. Total RNA was extracted from cells using the RNeasy Mini Kit from Qiagen (Valencia, CA, USA). Eluted RNA was treated with DNaseI (NEB, Ipswich, MA, USA) before cleaning and purifying the RNA using an RNeasy Mini Kit column (Qiagen, Valencia, CA, USA) according to the manufacturers recommendations. RNA quality was assessed by agarose gel electrophoresis and absence of contaminating DNA was confirmed by PCR with primers to the large tandem repeat DXZ4 [86]. Single stranded cDNA was prepared using the High Capacity cDNA Reverse Transcription Kit (Invitrogen, Grand Island, NY, USA: Cat. No. 4368814). The cDNA was then provided to the FSU-Nimblegen Microarray Facility for labeling, hybridization, and washing. Probes were hybridized onto a Human Expression $12 \times 135 \mathrm{~K}$ array (Roche-Nimblegen, Indianapolis, IN, USA: 05543789001). Hybridization data was analyzed using DNA ArrayStar version 4 (DNASTAR, Madison, WI, USA). The three replicates of RPE1 were compared to one another. Transcript targets were included if their confidence level was $\geq 95 \%$ based on a $\mathrm{p}$-value of $\mathrm{p}<0.05$. This was done for each of the mutant clones. Replicate sets of each of the mutants were compared to the RPE1 replicate set. In order to be included, greater than two fold change in expression was required, with a confidence level of $\geq 95 \%$ based on a $\mathrm{p}$-value of $\mathrm{p}<0.05$. Additionally, transcript targets that did not meet these criteria in all three of the independent mutants were excluded. Microarray expression data has been submitted to the Gene Expression Omnibus, and has been assigned accession number GSE47390.

\section{Quantitative RT-PCR Analysis}

All qRT-PCR was performed on a CFX96 (Biorad, Hercules, CA, USA) using EvaGreen 2X qPCR Mastermix (ABM, Richmond, BC, Canada: Cat No. MastermixS). The two-step amplification cycle began with a $10 \mathrm{mi}-$ nute incubation at $95 \mathrm{C}$, followed by 40 cycles of 15 seconds at $95 \mathrm{C}$ and 30 seconds at $60 \mathrm{C}$. Fluorescence was measured at the end of each of the 40 cycles. Afterwards, a melt curve was generated by measuring the fluorescence after heating for 5 seconds in $0.5 \mathrm{C}$ increments, beginning at $65 \mathrm{C}$ and ending at 95C. qRT-PCR analysis of $B A Z 1 B$ used Quantitect primer assay QT00072044 along with GAPDH assay QT01192646. Both were obtained from Qiagen (Valencia, CA, USA).

\section{Standard RT-PCR Analysis}

Standard RT-PCR was performed using OneTaq (NEB, Ipswich, MA, USA). The cDNA samples were denatured at $94^{\circ} \mathrm{C}$ for 30 seconds, amplified through 40 cycles of 30 seconds at $94^{\circ} \mathrm{C}, 30$ seconds at $58^{\circ} \mathrm{C}$, and 30 seconds at $68^{\circ} \mathrm{C}$, and finally held at $15^{\circ} \mathrm{C}$. Details on the primers used can be found in Additional file 7 .

\section{Additional files}

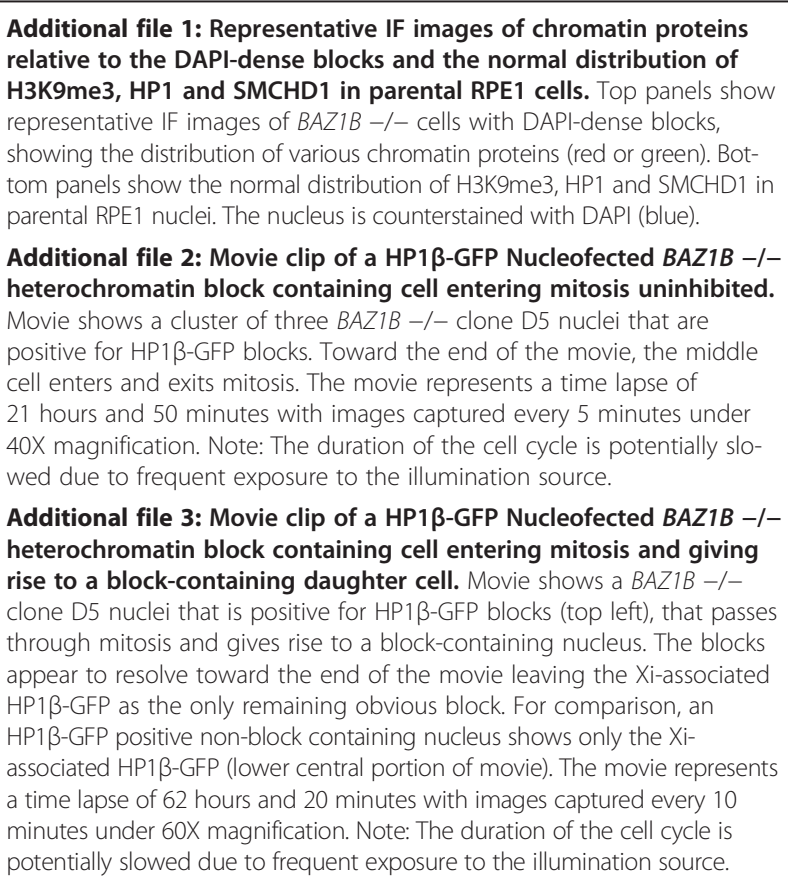

Additional file 1: Representative IF images of chromatin proteins relative to the DAPI-dense blocks and the normal distribution of representative IF images of BAZ1B-/- cells with DAPI-dense blocks, showing the distribution of various chromatin proteins (red or green). Bottom panels show the normal distribution of H3K9me3, HP1 and SMCHD1 in parental RPE1 nuclei. The nucleus is counterstained with DAPI (blue).

Additional file 2: Movie clip of a HP1 $\beta$-GFP Nucleofected BAZ1B -/heterochromatin block containing cell entering mitosis uninhibited. Movie shows a cluster of three BAZ1B -/- clone D5 nuclei that are positive for HP1ß-GFP blocks. Toward the end of the movie, the middle cell enters and exits mitosis. The movie represents a time lapse of 21 hours and 50 minutes with images captured every 5 minutes under 40X magnification. Note: The duration of the cell cycle is potentially slowed due to frequent exposure to the illumination source. rise to a block-containing daughter cell. Movie shows a BAZ1B -/clone D5 nuclei that is positive for HP1ß-GFP blocks (top left), that passes through mitosis and gives rise to a block-containing nucleus. The blocks appear to resolve toward the end of the movie leaving the Xi-associated a time lapse of 62 hours and 20 minutes with images captured every 10 potentially slowed due to frequent exposure to the illumination source. 


\begin{abstract}
Additional file 4: Movie clip of a HP1 $\beta$-GFP Nucleofected BAZ1B -/heterochromatin block containing cell showing the static nature of the block positions in the nucleus. Movie shows a BAZ1B - /- clone D5 nuclei that is positive for HP1ß-GFP blocks, that develops intense blocks that show no obvious movement despite the mobility of the cell. The movie represents a time lapse of 62 hours and 20 minutes with images captured every 10 minutes under 60X magnification. Note: The duration of the cell cycle is potentially slowed due to frequent exposure to the illumination source.
\end{abstract}

\section{Additional file 5: Excel file listing genes that consistently change} expression by $>2$-fold in $B A Z 1 B-/$ - cells. File contains tabs for all data combined, as well as gene expression for each chromosome individually.

Additional file 6: Excel file listing genes that consistently change expression by $>2$-fold in $B A Z 1 B+/-$ cells that are shared with $B A Z 1 B-/-$ cells.

Additional file 7: Table listing all oligonucleotides used in this study.

\section{Abbreviations}

BAZ1B: Bromodomain adjacent to zinc finger domain 1B; BRCA1: Breast cancer 1 gene; DAPI: 4',6-diamidino-2-phenylindole; FACS: Fluorescence activated cell sorting; FISH: Fluorescence in situ hybridization; GFP: Green fluorescent protein; Y-H2A.X: Histone H2A.X phosphorylated at serine 139; H2A.X: Histone H2A variant X; H2A.X-Y142ph: H2A.X phosphorylated at tyrosine 142; H3K4me2: Histone H3 dimethylated at lysine 4; H3K9Ac: Histone $\mathrm{H3}$ acetylated at lysine 9; $\mathrm{H3}$ K9me3: Histone H3 trimethylated at lysine 9; H3K27me3: Histone H3 trimethylated at lysine 27; H3S10ph: Histone H3 phosphorylated at serine 10; HA: Homology arm; HP1: Heterochromatin protein 1; IF: Immunofluorescence; mH2A1: macroH2A1; NHEJ: Non homologous end joining; NOR: Nucleolar organizing region; ORF: Open reading frame; PCNA: Proliferating cell nuclear antigen; PCR: Polymerase chain reaction; PML: Promyelocytic leukemia; QRT-PCR: Quantitative real time polymerase chain reaction; rDNA: Ribosomal deoxyribonucleic acid; RNAi: RNA interference; RPE1: hTERT-Retinal pigment epithelial; RT-PCR: Real time polymerase chain reaction; SEPT: Synthetic exon promoter trap; SMCHD1: Structural maintenance of chromosomes flexible hinge domain containing 1; SNF2H: Sucrose nonfermenting 2 homolog; Try142: Tyrosine 142; WBS: Williams-Beuren syndrome; WICH: WSTF-ISWI chromatin remodeling complex; WSTF: Williams syndrome transcription factor; XCI: X chromosome inactivation; Xi: Inactive X chromosome; XIST: X-inactive specific transcript; ZFN: Zinc finger nuclease.

\section{Competing interests}

Both authors declare they have no competing interests.

\section{Authors' contributions}

Conceived and designed the experiments: AECC, BPC. Performed the experiments: AECC, BPC. Analyzed the data: AECC, BPC. Contributed reagents/materials/analysis tools: AECC, BPC. Wrote the paper: AECC, BPC. Both authors read and approved the final manuscript.

\section{Acknowledgements}

We are grateful to Dr. P. Finelli (University of Milan, Italy) for provision of the PAC clone RP5-1174A5. We are grateful to Fred Bunz (Johns Hopkins University School of Medicine) for the PSEPT vector. We would like to thank Dr. J. Cochran (University of South Florida) for constructive comments on the manuscript, Dr. R. Didier (Florida State University, College of Medicine) for assistance with FACS analysis and live cell imaging, and Dr. S. Miller (Florida State University) for assistance with microarray hybridization. We are grateful to Sunny Das (Florida State University) for assistance with the microarray analysis. This work was supported by the National Institutes of Health

[GM073120 to B.P.C.], and the Florida State University FYAP program.

Received: 12 June 2013 Accepted: 26 October 2013

Published: 29 October 2013

\section{References}

1. Lyon MF: Gene action in the X-chromosome of the mouse (Mus musculus L.). Nature 1961, 190:372-373.
2. Lee JT, Bartolomei MS: X-inactivation, imprinting, and long noncoding RNAs in health and disease. Cell 2013, 152:1308-1323.

3. Chadwick BP, Willard HF: Multiple spatially distinct types of facultative heterochromatin on the human inactive $\mathrm{X}$ chromosome. Proc Natl Acad SCi U S A 2004, 101:17450-17455.

4. Boggs BA, Cheung P, Heard E, Spector DL, Chinault AC, Allis CD: Differentially methylated forms of histone $\mathrm{H} 3$ show unique association patterns with inactive human X chromosomes. Nat Genet 2002, 30:73-76.

5. Peters AH, Mermoud JE, O'Carroll D, Pagani M, Schweizer D, Brockdorff N, Jenuwein T: Histone H3 lysine 9 methylation is an epigenetic imprint of facultative heterochromatin. Nat Genet 2002, 30:77-80.

6. Chadwick BP, Willard HF: Chromatin of the Barr body: histone and nonhistone proteins associated with or excluded from the inactive $\mathrm{X}$ chromosome. Hum Mol Genet 2003, 12:2167-2178.

7. Plath K, Fang J, Mlynarczyk-Evans SK, Cao R, Worringer KA, Wang H, de la Cruz CC, Otte AP, Panning B, Zhang Y: Role of histone H3 lysine 27 methylation in X inactivation. Science 2003, 300:131-135.

8. Silva J, Mak W, Zvetkova I, Appanah R, Nesterova TB, Webster Z, Peters AH, Jenuwein T, Otte AP, Brockdorff N: Establishment of histone h3 methylation on the inactive $\mathrm{x}$ chromosome requires transient recruitment of eed-enx1 polycomb group complexes. Dev Cell 2003, 4:481-495.

9. Chadwick BP, Willard HF: Histone H2A variants and the inactive $X$ chromosome: identification of a second macroH2A variant. Hum Mol Genet 2001, 10:1101-1103.

10. Costanzi C, Pehrson JR: Histone macroH2A1 is concentrated in the inactive X chromosome of female mammals. Nature 1998, 393:599-601.

11. Costanzi C, Pehrson JR: MacroH2A2, a new member of the MacroH2A core histone family. J Biol Chem 2001, 276:21776-21784.

12. Brockdorff N, Ashworth A, Kay GF, McCabe VM, Norris DP, Cooper PJ, Swift $\mathrm{S}$, Rastan S: The product of the mouse Xist gene is a $15 \mathrm{~kb}$ inactive $X$-specific transcript containing no conserved ORF and located in the nucleus. Cell 1992, 71:515-526.

13. Brown $C J$, Hendrich BD, Rupert JL, Lafreniere RG, Xing Y, Lawrence J, Willard HF: The human XIST gene: analysis of a $17 \mathrm{~kb}$ inactive X-specific RNA that contains conserved repeats and is highly localized within the nucleus. Cell 1992, 71:527-542.

14. Clemson CM, MCNeil JA, Willard HF, Lawrence JB: XIST RNA paints the inactive $\mathrm{X}$ chromosome at interphase: evidence for a novel RNA involved in nuclear/chromosome structure. J Cell Biol 1996, 132:1-17.

15. Culver-Cochran AE, Chadwick BP: The WSTF-ISWI chromatin remodeling complex transiently associates with the human inactive $\mathrm{X}$ chromosome during late S-phase prior to BRCA1 and gamma-H2AX. PLoS One 2012, 7:e50023.

16. Bozhenok L, Wade PA, Varga-Weisz P: WSTF-ISWI chromatin remodeling complex targets heterochromatic replication foci. EMBO J 2002, 21:2231-2241.

17. Poot RA, Bozhenok L, van den Berg DL, Hawkes N, Varga-Weisz PD: Chromatin remodeling by WSTF-ISWI at the replication site: opening a window of opportunity for epigenetic inheritance? Cell Cycle 2005, 4:543-546.

18. Poot RA, Bozhenok L, van den Berg DL, Steffensen S, Ferreira F, Grimaldi $M$ Gilbert N, Ferreira J, Varga-Weisz PD: The Williams syndrome transcription factor interacts with PCNA to target chromatin remodelling by ISWI to replication foci. Nat Cell Biol 2004, 6:1236-1244.

19. Lu X, Meng X, Morris CA, Keating MT: A novel human gene, WSTF, is deleted in Williams syndrome. Genomics 1998, 54:241-249.

20. Cavellan E, Asp P, Percipalle P, Farrants AK: The WSTF-SNF2h chromatin remodeling complex interacts with several nuclear proteins in transcription. J Biol Chem 2006, 281:16264-16271.

21. Xiao A, Li H, Shechter D, Ahn SH, Fabrizio LA, Erdjument-Bromage H, IshibeMurakami S, Wang B, Tempst P, Hofmann K, et al: WSTF regulates the H2A. $\mathrm{X}$ DNA damage response via a novel tyrosine kinase activity. Nature 2009, 457:57.

22. Percipalle P, Fomproix N, Cavellan E, Voit R, Reimer G, Kruger T, Thyberg J, Scheer U, Grummt I, Farrants AK: The chromatin remodelling complex WSTF-SNF2h interacts with nuclear myosin 1 and has a role in RNA polymerase I transcription. EMBO Rep 2006, 7:525-530.

23. Cook PJ, Ju BG, Telese F, Wang X, Glass CK, Rosenfeld MG: Tyrosine dephosphorylation of $\mathrm{H} 2 \mathrm{AX}$ modulates apoptosis and survival decisions. Nature 2009, 458:591-596.

24. Stucki M: Histone H2A.X Tyr142 phosphorylation: a novel sWItCH for apoptosis? DNA Repair (Amst) 2009, 8:873-876.

25. Stromme P, Bjornstad PG, Ramstad K: Prevalence estimation of Williams syndrome. J Child Neurol 2002, 17:269-271. 
26. Perez Jurado LA, Peoples R, Kaplan P, Hamel BC, Francke U: Molecular definition of the chromosome 7 deletion in Williams syndrome and parent-of-origin effects on growth. Am J Hum Genet 1996, 59:781-792.

27. Valero MC, de Luis O, Cruces J, Perez Jurado LA: Fine-scale comparative mapping of the human $7 q 11.23$ region and the orthologous region on mouse chromosome 5G: the low-copy repeats that flank the WilliamsBeuren syndrome deletion arose at breakpoint sites of an evolutionary inversion(s). Genomics 2000, 69:1-13.

28. Bayes M, Magano LF, Rivera N, Flores R, Perez Jurado LA: Mutational mechanisms of Williams-Beuren syndrome deletions. Am J Hum Genet 2003, 73:131-151.

29. DeSilva U, Elnitski L, Idol JR, Doyle JL, Gan W, Thomas JW, Schwartz S, Dietrich NL, Beckstrom-Sternberg SM, McDowell JC, et al: Generation and comparative analysis of approximately $3.3 \mathrm{Mb}$ of mouse genomic sequence orthologous to the region of human chromosome $7 q 11.23 \mathrm{im}$ plicated in Williams syndrome. Genome Res 2002, 12:3-15.

30. Merla G, Ucla C, Guipponi M, Reymond A: Identification of additional transcripts in the Williams-Beuren syndrome critical region. Hum Genet 2002, 110:429-438.

31. Peoples RJ, Cisco MJ, Kaplan P, Francke U: Identification of the WBSCR9 gene, encoding a novel transcriptional regulator, in the Williams-Beuren syndrome deletion at 7q11.23. Cytogenet Cell Genet 1998, 82:238-246.

32. Bellugi U, Bihrle A, Jernigan T, Trauner D, Doherty S: Neuropsychological, neurological, and neuroanatomical profile of Williams syndrome. Am J Med Genet Supp/ 1990, 6:115-125.

33. Burn J: Williams syndrome. J Med Genet 1986, 23:389-395.

34. Morris CA, Demsey SA, Leonard CO, Dilts C, Blackburn BL: Natural history of Williams syndrome: physical characteristics. J Pediatr 1988, 113:318-326.

35. Cus R, Maurus D, Kuhl M: Cloning and developmental expression of WSTF during Xenopus laevis embryogenesis. Gene Expr Patterns 2006, 6:340-346.

36. Yoshimura K, Kitagawa H, Fujiki R, Tanabe M, Takezawa S, Takada I, Yamaoka I, Yonezawa $\mathrm{M}$, Kondo T, Furutani $\mathrm{Y}$, et al: Distinct function of 2 chromatin remodeling complexes that share a common subunit, Williams syndrome transcription factor (WSTF). Proc Natl Acad Sci U S A 2009, 106:9280-9285.

37. Bodnar AG, Ouellette M, Frolkis M, Holt SE, Chiu CP, Morin GB, Harley CB, Shay JW, Lichtsteiner S, Wright WE: Extension of life-span by introduction of telomerase into normal human cells. Science 1998, 279:349-352.

38. Carroll D: Genome engineering with zinc-finger nucleases. Genetics 2011, 188:773-782.

39. Topaloglu O, Hurley PJ, Yildirim O, Civin Cl, Bunz F: Improved methods for the generation of human gene knockout and knockin cell lines. Nucleic Acids Res 2005, 33:e158.

40. Lieber MR: The mechanism of double-strand DNA break repair by the nonhomologous DNA end-joining pathway. Annu Rev Biochem 2010, 79:181-211.

41. Barr ML, Bertram EG: A morphological distinction between neurones of the male and female, and the behaviour of the nucleolar satellite during accelerated nucleoprotein synthesis. Nature 1949, 163:676-677.

42. Casas-Delucchi CS, Brero A, Rahn HP, Solovei I, Wutz A, Cremer T, Leonhardt $\mathrm{H}$, Cardoso MC: Histone acetylation controls the inactive $X$ chromosome replication dynamics. Nat Commun 2011, 2:222.

43. Chadwick BP, Willard HF: Cell cycle-dependent localization of macroH2A in chromatin of the inactive $X$ chromosome. J Cell Biol 2002, 157:1113-1123.

44. Jeppesen $P$, Turner BM: The inactive $\mathbf{X}$ chromosome in female mammals is distinguished by a lack of histone $\mathrm{H} 4$ acetylation, a cytogenetic marker for gene expression. Cell 1993, 74:281-289.

45. Chadwick BP, Lane TF: BRCA1 associates with the inactive $X$ chromosome in late S-phase, coupled with transient $\mathrm{H} 2 \mathrm{AX}$ phosphorylation. Chromosoma 2005, 114:432-439.

46. Ito T, Levenstein ME, Fyodorov DV, Kutach AK, Kobayashi R, Kadonaga J: ACF consists of two subunits, Acf1 and ISWI, that function cooperatively in the ATPdependent catalysis of chromatin assembly. Genes Dev 1999, 13:1529-1539.

47. Strohner R, Nemeth A, Jansa P, Hofmann-Rohrer U, Santoro R, Langst G, Grummt I: NoRC-a novel member of mammalian ISWI-containing chromatin remodeling machines. EMBO J 2001, 20:4892-4900.

48. Tsukiyama T, Daniel C, Tamkun J, Wu C: ISWI, a member of the SWI2/SNF2 ATPase family, encodes the $140 \mathrm{kDa}$ subunit of the nucleosome remodeling factor. Cell 1995, 83:1021-1026.

49. Varga-Weisz PD, Wilm M, Bonte E, Dumas K, Mann M, Becker PB: Chromatin-remodelling factor CHRAC contains the ATPases ISWI and topoisomerase II. Nature 1997, 388:598-602.
50. Guenatri M, Bailly D, Maison C, Almouzni G: Mouse centric and pericentric satellite repeats form distinct functional heterochromatin. J Cell Biol 2004, 166:493-505.

51. Narita M, Narita M, Krizhanovsky V, Nunez S, Chicas A, Hearn SA, Myers MP, Lowe SW: A novel role for high-mobility group a proteins in cellular senescence and heterochromatin formation. Cell 2006, 126:503-514.

52. Saunders WS, Chue C, Goebl M, Craig C, Clark RF, Powers JA, Eissenberg JC, Elgin SC, Rothfield NF, Earnshaw WC: Molecular cloning of a human homologue of Drosophila heterochromatin protein HP1 using anti-centromere autoantibodies with anti-chromo specificity. J Cell Sci 1993, 104:573-582.

53. Singh PB, Miller JR, Pearce J, Kothary R, Burton RD, Paro R, James TC, Gaunt $\mathrm{SJ}$ : A sequence motif found in a Drosophila heterochromatin protein is conserved in animals and plants. Nucleic Acids Res 1991, 19:789-794.

54. Ye Q, Worman HJ: Interaction between an integral protein of the nuclear envelope inner membrane and human chromodomain proteins homologous to Drosophila HP1. J Biol Chem 1996, 271:14653-14656.

55. Bannister AJ, Zegerman P, Partridge JF, Miska EA, Thomas JO, Allshire RC, Kouzarides T: Selective recognition of methylated lysine 9 on histone $\mathrm{H} 3$ by the HP1 chromo domain. Nature 2001, 410:120-124.

56. Jacobs SA, Taverna SD, Zhang Y, Briggs SD, Li J, Eissenberg JC, Allis D, Khorasanizadeh S: Specificity of the HP1 chromo domain for the methylated N-terminus of histone H3. EMBO J 2001, 20:5232-5241.

57. Lachner M, O'Carroll D, Rea S, Mechtler K, Jenuwein T: Methylation of histone H3 lysine 9 creates a binding site for HP1 proteins. Nature 2001, 410:116-120.

58. Nozawa RS, Nagao K, Igami KT, Shibata S, Shirai N, Nozaki N, Sado T, Kimura $\mathrm{H}$, Obuse $\mathrm{C}$ : Human inactive $\mathrm{X}$ chromosome is compacted through a PRC2-independent SMCHD1-HBiX1 pathway. Nat Struct Mol Biol 2013, 20:566-573.

59. Minc E, Allory Y, Worman HJ, Courvalin J-C, Buendia B: Localization and phosphorylation of HP1 proteins during the cell cycle in mammalian cells. Chromosoma 1999, 108:220-234.

60. Earnshaw WC, Rothfield N: Identification of a family of human centromere proteins using autoimmune sera from patients with scleroderma. Chromosoma 1985, 91:313-321

61. Warburton PE, Cooke CA, Bourassa S, Vafa O, Sullivan BA, Stetten G, Gimelli G, Warburton D, Tyler-Smith C, Sullivan KF, et al: Immunolocalization of CENP-A suggests a distinct nucleosome structure at the inner kinetochore plate of active centromeres. Curr Biol 1997, 7:901-904.

62. Barnett $C$, Krebs JE: WSTF does it all: a multifunctional protein in transcription, repair, and replication. Biochem Cell Biol 2011, 89:12-23.

63. Lallemand-Breitenbach V, de The H: PML nuclear bodies. Cold Spring Harb Perspect Biol 2010, 2:a000661

64. Nishitani H, Taraviras S, Lygerou Z, Nishimoto T: The human licensing factor for DNA replication Cdt1 accumulates in G1 and is destabilized after initiation of S-phase. J Biol Chem 2001, 276:44905-44911.

65. Wohlschlegel JA, Dwyer BT, Dhar SK, Cvetic C, Walter JC, Dutta A: Inhibition of eukaryotic DNA replication by geminin binding to Cdt1. Science 2000, 290:2309-2312.

66. Celis JE, Celis A: Cell cycle-dependent variations in the distribution of the nuclear protein cyclin proliferating cell nuclear antigen in cultured cells: subdivision of S phase. Proc Natl Acad Sci U S A 1985, 82:3262-3266.

67. Leonhardt H, Rahn HP, Weinzierl P, Sporbert A, Cremer T, Zink D, Cardoso MC: Dynamics of DNA replication factories in living cells. J Cell Biol 2000, 149:271-280.

68. Hendzel MJ, Wei Y, Mancini MA, Hooser AV, Ranalli T, Brinkley BR, BazettJones DP, Allis CD: Mitosis-specific phosphorylation of histone $\mathrm{H} 3$ intiates primarily within pericentromeric heterochromatin during $\mathrm{G} 2$ and spreads in an ordered fashion coincident with mitotic chromosome condensation. Chromosoma 1997, 106:348-360.

69. El-Amraoui A, Petit C: Cadherins as targets for genetic diseases. Cold Spring Harb Perspect Biol 2010, 2:a003095.

70. Dallosso AR, Hancock AL, Szemes M, Moorwood K, Chilukamarri L, Tsai HH, Sarkar A, Barasch J, Vuononvirta R, Jones C, et al: Frequent long-range epigenetic silencing of protocadherin gene clusters on chromosome $5 q 31$ in Wilms' tumor. PLoS Genet 2009, 5:e1000745.

71. Dallosso AR, Oster B, Greenhough A, Thorsen K, Curry TJ, Owen C, Hancock $A L$, Szemes $M$, Paraskeva C, Frank $M$, et al: Long-range epigenetic silencing of chromosome 5 q31 protocadherins is involved in early and late stages of colorectal tumorigenesis through modulation of oncogenic pathways. Oncogene 2012, 31:4409-4419. 
72. Wang J, Mager J, Chen Y, Schneider E, Cross JC, Nagy A, Magnuson T: Imprinted $\mathrm{X}$ inactivation maintained by a mouse Polycomb group gene. Nat Genet 2001, 28:371-375.

73. Kalantry S, Millis KC, Yee D, Otte AP, Panning B, Magnuson T: The Polycomb group protein Eed protects the inactive $X$-chromosome from differentiation-induced reactivation. Nat Cell Biol 2006, 8:195-202.

74. Mise N, Sado T, Tada M, Takada S, Takagi N: Activation of the inactive X chromosome induced by cell fusion between a murine EC and female somatic cell accompanies reproducible changes in the methylation pattern of the Xist gene. Exp Cell Res 1996, 223:193-202.

75. Sado T, Tada T, Takagi N: Mosaic methylation of Xist gene before chromosome inactivation in undifferentiated female mouse embryonic stem and embryonic germ cells. Dev Dyn 1996, 205:421-434.

76. Takagi N: De novo X-chromosome inactivation in somatic hybrid cells between the XO mouse embryonal carcinoma cell and XY rat lymphocyte. Exp Cell Res 1983, 145:397-404.

77. Takagi $\mathrm{N}$ : Variable $\mathbf{X}$ chromosome inactivation patterns in near-tetraploid murine EC $x$ somatic cell hybrid cells differentiated in vitro. Genetica 1993, 88:107-117.

78. Takahashi K, Yamanaka S: Induction of pluripotent stem cells from mouse embryonic and adult fibroblast cultures by defined factors. Cell 2006, 126:663-676.

79. van Koningsbruggen S, Gierlinski M, Schofield P, Martin D, Barton GJ, Ariyurek Y, den Dunnen JT, Lamond Al: High-resolution whole-genome sequencing reveals that specific chromatin domains from most human chromosomes associate with nucleoli. Mol Biol Cell 2010, 21:3735-3748.

80. Zhang LF, Huynh KD, Lee JT: Perinucleolar targeting of the inactive $X$ during $S$ phase: evidence for a role in the maintenance of silencing. Cell 2007, 129:693-706.

81. Chadwick BP, Willard HF: A novel chromatin protein, distantly related to histone $\mathrm{H} 2 \mathrm{~A}$, is largely excluded from the inactive $\mathrm{X}$ chromosome. I Cell Biol 2001, 152:375-384

82. Harlow E, Lane D: Using Antibodies: A Laboratory Manual. Cold Spring Harbor, NY: Cold Spring Harbor Laboratory Press; 1999.

83. McLaughlin CR, Chadwick BP: Characterization of DXZ4 conservation in primates implies important functional roles for CTCF binding, array expression and tandem repeat organization on the $\mathrm{X}$ chromosome. Genome Biol 2011, 12:R37.

84. Grimes BR, Babcock J, Rudd MK, Chadwick B, Willard HF: Assembly and characterization of heterochromatin and euchromatin on human artificial chromosomes. Genome Biol 2004, 5:R89.

85. Alekseev OM, Bencic DC, Richardson RT, Widgren EE, O'Rand MG: Overexpression of the Linker histone-binding protein tNASP affects progression through the cell cycle. J Biol Chem 2003, 278:8846-8852.

86. Chadwick BP: DXZ4 chromatin adopts an opposing conformation to that of the surrounding chromosome and acquires a novel inactive $X$-specific role involving CTCF and antisense transcripts. Genome Res 2008, 18:1259-1269.

doi:10.1186/1471-2164-14-740

Cite this article as: Culver-Cochran and Chadwick: Loss of WSTF results in spontaneous fluctuations of heterochromatin formation and resolution, combined with substantial changes to gene expression. BMC Genomics 2013 14:740.

\section{Submit your next manuscript to BioMed Central and take full advantage of:}

- Convenient online submission

- Thorough peer review

- No space constraints or color figure charges

- Immediate publication on acceptance

- Inclusion in PubMed, CAS, Scopus and Google Scholar

- Research which is freely available for redistribution 